\begin{tabular}{llllll} 
& ISRA (India) $=\mathbf{3 . 1 1 7}$ & SIS (USA) $=\mathbf{0 . 9 1 2}$ & ICV (Poland) & $\mathbf{= 6 . 6 3 0}$ \\
Impact Factor: & ISI (Dubai, UAE) $=\mathbf{0 . 8 2 9}$ & PUHЦ (Russia) $=\mathbf{0 . 1 5 6}$ & PIF (India) & $=\mathbf{1 . 9 4 0}$ \\
& GIF (Australia) $=\mathbf{0 . 5 6 4}$ & ESJI (KZ) & $\mathbf{8 . 7 1 6}$ & IBI (India) & $=\mathbf{4 . 2 6 0}$ \\
& JIF & $\mathbf{1 . 5 0 0}$ & SJIF (Morocco) $=\mathbf{5 . 6 6 7}$ & OAJI (USA) & $\mathbf{0 . 3 5 0}$ \\
\hline
\end{tabular}

\section{SOI: $1.1 /$ TAS $\quad$ DOI: $10.15863 /$ TAS International Scientific Journal Theoretical \& Applied Science}

p-ISSN: 2308-4944 (print) e-ISSN: 2409-0085 (online)

Year: $2019 \quad$ Issue: $06 \quad$ Volume: 74

Published: $30.06 .2019 \quad \underline{\text { http://T-Science.org }}$

SECTION 2. Applied mathematics.

Mathematical modeling.
QR - Issue

QR - Article

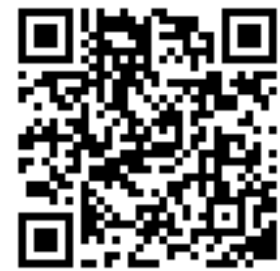

Noncommercial joint-stock company Kazakh national agrarian university Corresponding Member of International Academy of Theoretical and Applied Sciences (USA), Professor, Candidate of physics and mathematical sciences, Department «Information technologies and automatiization», Kazakhstan sapagtu@mail.ru

\title{
COEFFICIENTS OF REGRESSION, CONTAINING MATHEMATICALLY INTRODUCED AND COGNITIVELY EXTRACTABLED KNOWLEDGE
}

\begin{abstract}
The article developed a variant of the Inverse Model of Multiple Linear Regression Analysis (OM $M L R A$ with given regression coefficients $\left.=\left(\beta_{1}, \ldots, \beta_{n-1}\right)^{T}, Z^{+}{ }_{2}=Z^{+}{ }_{1} \boldsymbol{\beta}, Z^{+}{ }_{m n}=\left[Z^{+}{ }_{1}\right\rfloor Z^{+}{ }_{2}\right]$, containing mathematically int of produced and cognitively extracted knowledge With the use of equalities from Inverse Model the Principal Component Analysis (IM PCA) solved a New Inverse Generalized Problem of Multiple Linear Regression Analysis (IGM MLRA) of statistical modeling of $n$ - 1 correlated $z$-variables, $1 z$-variable: $\left.Z^{+}{ }_{m n}=\left[Z^{+}{ }_{1}\right] Z^{+}{ }_{2}\right], Z^{+}{ }_{2}=Z^{+}{ }_{1} \boldsymbol{\beta}$, satisfying all equations and relations in DM MLRA: $\left(Z^{+}{ }_{1}, Z^{+}{ }_{2}\right)=>\left(R^{-1}{ }_{11}, R_{12}, \boldsymbol{\beta}\right)$. Simulated $\left(\boldsymbol{\beta}, C^{+}{ }_{11}\right)$--samples $\left.Z^{+}{ }_{m n}=\left[Z^{+}{ }_{1}\right] Z^{+}{ }_{2}\right]$, $Z^{+}{ }_{2}=Z^{+}{ }_{1} \beta$, in the presence of a partition, and the extraction of knowledge - without partitioning the set of $z-$ variables. The input parameters of the IGP of MLRA are the vector $\beta=\left(\beta_{1}, \ldots, \beta_{n-1}\right)^{T}$ and the matrix of eigenvectors $C^{+}{ }_{11}$. The numerical algorithms were tested using the example of modeling a multidimensional $\left(\boldsymbol{\beta}, C^{+}{ }_{11}\right)$-sample of $z$ - variables (with $n=6$ ). The designated vector $\boldsymbol{\beta}=\left(\beta_{1} \ldots, \beta_{6}\right)^{T}$ regression coefficients was used. A description of the reverse process of extracting the knowledge entered using cognitive modeling will be published in another article.

Key words: mathematically introduced, cognitively extracted knowledge.

Language: Russian

Citation: Zhanatauov, S. U. (2019). Coefficients of regression, containing mathematically introduced and cognitively extractabled knowledge. ISJ Theoretical \& Applied Science, 06 (74), 613-622.

Soi: http://s-o-i.org/1.1/TAS-06-74-75 Doi: croskef https://dx.doi.org/10.15863/TAS.2019.06.74.75

\section{КОЭФФИЦИЕНТЫ РЕГРЕССИИ, СОДЕРЖАЩИЕ МАТЕМАТИЧЕСКИ ВВЕДЕННЫЕ И КОГНИТИВНО ИЗВЛЕКАЕМЫЕ ЗНАНИЯ}

Аннотация: В статье разработан вариант Обратной Модели Множественного Линейного Регрессионного Анализа (ОМ МЛРА с заданными значениями коэффициентов регрессии $\boldsymbol{\beta}=\left(\beta_{1}, \ldots, \beta_{n}\right.$ $\left.\left.{ }_{1}\right)^{T}, Z^{+}{ }_{2}=Z^{+}{ }_{1} \boldsymbol{\beta}, Z^{+}{ }_{m n}=\left[Z^{+}{ }_{1}\right] Z^{+}{ }_{2}\right]$, содержащих математически введенные и когнитивно извлекаемые знания. $C$ применением равенств из Обратной Модели Главных Компонент (ОМ ГК) решена новая Обратная Обобщенная Задача Множественного Линейного Регрессионного Анализа (ООЗ МЛРА) статистического моделирования значений $n-1$ коррелированных z-переменных, 1 z-переменной: $\left.Z^{+}{ }_{m n}=\left[Z^{+}{ }_{1}\right] Z^{+}{ }_{2}\right], Z^{+}{ }_{2}=Z^{+}{ }_{1} \boldsymbol{\beta}$, удовлетворяюших всем уравнениям и соотношениям ПМ МЛРА: $\left(Z^{+}{ }_{1}, Z^{+}{ }_{2}\right)=>\left(R^{-1}{ }_{11}, R_{12}, \boldsymbol{\beta}\right)$. Моделируются $\left(\boldsymbol{\beta}, C^{+}{ }_{11}\right)$-выборки $\left.Z^{+}{ }_{m n}=\left[Z^{+}{ }_{1}\right\rfloor Z^{+}{ }_{2}\right], Z^{+}{ }_{2}=Z^{+}{ }_{1} \boldsymbol{\beta}$, при наличии разбиения, а извлечение знаний - без разбиения множества $z$-переменных. Входными параметрами ООЗ МЛРА являются вектор $\boldsymbol{\beta}=\left(\beta_{1}, \ldots, \beta_{n-1}\right)^{T}$ и матрица собственных векторов $C^{+}{ }_{11}$. Численные алгоритмы апробированы на примере моделирования многомерной $\left(\boldsymbol{\beta}, C^{+}{ }_{11}\right)$-выборки z-переменных (при $\left.n=6\right)$. Использован назначенный вектор $\boldsymbol{\beta}=\left(\beta_{1} \ldots, \beta_{6}\right)^{T}$ регрессионных коэффициентов. Описание обратного процесса извлечения введенных знаний с применением когнитивного моделирования будет опубликовано в другой статье.
\end{abstract}

Ключевые слова: математически введенные знания, когнитивно извлекаемые знания. 


\begin{tabular}{|c|c|c|c|c|c|c|}
\hline \multirow{4}{*}{ Impact Factor: } & ISRA (India) & $=3.117$ & SIS (USA) & $=0.912$ & ICV (Poland) & $=6.630$ \\
\hline & ISI (Dubai, UAI & $=0.829$ & РИНЦ (Russia & $=0.156$ & PIF (India) & $=1.940$ \\
\hline & GIF (Australia) & $=0.564$ & ESJI (KZ) & $=8.716$ & IBI (India) & $=4.260$ \\
\hline & JIF & $=1.500$ & SJIF (Morocce & $=5.667$ & OAJI (USA) & $=0.350$ \\
\hline
\end{tabular}

\section{Введение}

ОМ МЛРА предложена в [1] и применена в [2]. В Обратной Модели Множественного Линейного Анализа предполагается существование уравнения регрессии вида $\mathrm{z}_{\mathrm{n}}=\beta_{1} \mathrm{z}_{1}+\beta_{2} \mathrm{z}_{2}+\ldots+\beta_{\mathrm{n}-1} \mathrm{z}_{\mathrm{n}-1}$, где, в отличие от Прямой Задачи Множественного Линейного Регрессионного Анализа (ПМ МЛРА) известны значения $\beta_{1}, \ldots, \beta_{\mathrm{n}-1} \quad$ вектора $\quad \beta=\left(\beta_{1}, \ldots, \beta_{\mathrm{n}-1}\right)^{\mathrm{T}}$ регрессионных коэффициентов. Известную ПМ МЛРА[3] обозначим так: $Z_{m n}=\left[Z_{1} \mid Z_{2}\right]=>\left(R^{-1}{ }_{11}, R_{12}\right.$, $\beta)$. В ПМ МЛРА [3] решена ПЗ МЛРА, ее решение $\beta$ единственно и равно $\beta=\mathrm{R}^{-1}{ }_{11} \mathrm{R}_{12}$. Ранг матрицы $\mathrm{R}_{11}$ равен $\mathrm{n}-1$. Для каждого значения $\mathrm{z}_{\mathrm{n}}$ из реальной выборки и оценки ее значения из ПМ МЛА разность этих величин не равна нулю.

Мы считаем неудовлетворительным свойством единственность решение $\beta_{\mathrm{R}}=\mathrm{R}^{-1}{ }_{11} \mathrm{R}_{12}$. $\mathrm{B}$ OМ МЛРА [1] значения компонент $\beta_{1}, \ldots, \beta_{\mathrm{n}-1}$ вектора $\boldsymbol{\beta}=\left(\beta_{1}, \ldots, \beta_{\mathrm{n}-1}\right)^{\mathrm{T}}$ могут быть заменены на желаемых значения, а для них - смоделированы новые матрицы $\mathrm{Z}_{\mathrm{mn}}=\left[\mathrm{Z}_{1} \mid \mathrm{Z}_{2}\right]$ значений $\mathrm{n}$ zпеременных $\mathrm{Z}_{1}, \mathrm{Z}_{2}, \ldots, \mathrm{Z}_{\mathrm{n}}, \quad$ также точно удовлетворяют формуле

$\mathrm{z}_{\mathrm{n}}=\beta_{1} \mathrm{z}_{1}+\beta_{2} \mathrm{z}_{2}+\ldots+\beta_{\mathrm{n}-1} \mathrm{z}_{\mathrm{n}-1}$.

Вектор $\boldsymbol{\beta}=\left(\beta_{1}, \ldots, \beta_{\mathrm{n}-1}\right)^{\mathrm{T}}$ в ОМ МЛРА содержит математически введенные в его компоненты знания типа: единичное приращение регрессора $\mathrm{z}_{1}$ дает приращение $\beta_{1}$ к значению независимой переменной $\mathrm{Z}_{\mathrm{n}}$.

Необходимо дополнительно ввести в эти значения компонент вектора $\boldsymbol{\beta}=\left(\beta_{1}, \ldots, \beta_{\mathrm{n}-1}\right)^{\mathrm{T}}$ когнитивно извлекаемые знания. Это возможно, в частности, через матрицы $\mathrm{R}_{11}, \mathrm{R}_{12}$, математически определяющие вектор $\boldsymbol{\beta}=\left(\beta_{1}, \ldots, \beta_{\mathrm{n}-1}\right)^{\mathrm{T}}$.

Введем математические индикаторы наличия желаемых знаний в матрицу собственных векторов $\mathrm{C}_{11}$ корреляционной матрицы $\mathrm{R}_{11}$, a последующее извлечение введенных знаний проведем с применением соответствующей когнитивной модели, использующей математическую модель главных компонент [4-6]. Возможности соответствующих когнитивных моделей показаны в публикациях [4-6]. Здесь ниже изложим новую Обратную обобщенную Модель Множественной Линейной Регрессии, предназначенную для моделирования заданных значений коэффициентов регрессии. вектор $\boldsymbol{\beta}=\left(\beta_{1}, \ldots, \beta_{\mathrm{n}-1}\right)^{\mathrm{T}}$ коэффициентов регрессии содержат математически - через индикаторы наличия знаний [7] и когнитивно извлекаемые знания [4-6]. Преимущество ОМ МЛРА в том, что модельные значения $\mathrm{n}$ z-переменных точно удовлетворяют формуле $\mathrm{z}_{\mathrm{n}}=\beta_{1} \mathrm{Z}_{1}+\beta_{2} \mathrm{Z}_{2}+\ldots+\beta_{\mathrm{n}-1} \mathrm{Z}_{\mathrm{n}-1}$. Аддитивное случайное приращение $\alpha_{\mathrm{i}}, \mathrm{i}=1, \ldots, \mathrm{m}, \kappa$ значениям $Z_{\text {in }}$ (присущее ПМ МЛРА) в ОМ МЛРА придает вектору-решению $\left(\mathrm{z}_{\mathrm{i} 1}, \mathrm{z}_{\mathrm{i} 2}, \ldots, \mathrm{z}_{\mathrm{i}(\mathrm{n}-1)}, \mathrm{z}_{\mathrm{in}-1}\right)^{\mathrm{T}}$ нашей модельной выборки свойство ошибки предсказанного значения. Значениями этих ошибок в ОМ МЛРА можно управлять, что невозможно в ПМ МЛРА. Следовательно теоретическое решение ПМ МЛРА является одним из бесконечного множества теоретических решений ОМ МЛР - регрессионную модель вида $\mathrm{z}_{\mathrm{n}}=\beta_{1} \mathrm{Z}_{1}+\beta_{2} \mathrm{Z}_{2}+\ldots+\beta_{\mathrm{n}-1} \mathrm{z}_{\mathrm{n}-1}+\alpha$, где $\mathrm{z}_{1}, \mathrm{z}_{2}, \ldots, \mathrm{z}_{\mathrm{n}-1}$-набор объясняющих (независимых) переменных («регрессоров»), $\mathrm{Z}_{\mathrm{n}}$-переменная отклика (зависимая переменная), $\beta_{1}, \ldots, \beta_{\mathrm{n}-1}$-регрессионные коэффициенты, $\alpha$-свободный член. Эта модель отражает взаимосвязь между двумя или более объясняющими переменными и одной переменной отклика путем подгонки вышеприведенного линейного уравнения к стандартизованным значениям $\mathrm{z}$-переменных $\mathrm{z}_{\mathrm{ij}}=\left(\mathrm{x}_{\mathrm{ij}}^{0} \mathrm{X}^{\mathrm{cp}}{ }_{\mathrm{j}}\right) / \mathrm{s}_{\mathrm{j}}$. Законы распределения одномерных случайных величин $\xi_{1}, \xi_{2}, \ldots, \xi_{n}, \quad$ соответствующих $\mathrm{z}$-переменным $\mathrm{Z}_{1}, \mathrm{Z}_{2}, \ldots, \mathrm{Z}_{\mathrm{n}}$ не известны. Здесь $\mathrm{x}_{\mathrm{ij}}^{0} \mathrm{i}$-ое значение $\mathrm{j}$-го признака реального объекта, $\mathrm{x}^{\mathrm{cp}}=\left(\mathrm{x}^{0}{ }_{1 \mathrm{j}}+\ldots+\mathrm{x}_{\mathrm{mj}}^{0}\right) / \mathrm{m}$ - среднее арифметическое, $\mathrm{s}^{2}{ }_{\mathrm{j}}=\left(\mathrm{x}^{2}{ }_{1 \mathrm{j}}+\ldots+\mathrm{x}_{\mathrm{mj}}{ }_{\mathrm{j}}\right) / \mathrm{m}$ стандартное отклонение, $\mathrm{x}_{\mathrm{ij}}=\mathrm{x}_{\mathrm{ij}} \mathrm{x}^{\mathrm{cp}} \mathrm{j}_{\mathrm{j}}$ - отклонение от среднего значения $\mathrm{x}_{\mathrm{j}}^{\mathrm{c}}$. Стандартизованные значения $\mathrm{Z}_{\mathrm{n}}$ изменяются относительно значений $\mathrm{Z}_{1}, \mathrm{Z}_{2}, \ldots, \mathrm{Z}_{\mathrm{n}-1}$ с одинаковыми стандартными отклонениями, равными 1.

В соответствии с этим разбиением $\mathrm{Z}$ переменных $\mathrm{m}$ значений всех $\mathrm{n}$ z-переменных образует 2 подматрицы $Z_{1}, Z_{2}$ матрицы $Z_{m n}=\left[Z_{1} \mid Z_{2}\right]$ для m-на-n матрицы $Z_{\mathrm{mn}}$. Элементы столбцов (с номерами $\mathrm{j}=1, \ldots, n)$ матрицы $\mathrm{Z}_{\mathrm{mn}}$ центрированы выборочными средними и нормированы стандартными отклонениями: $\mathrm{z}_{\mathrm{ij}}=\left(\mathrm{x}^{0} \mathrm{ij}_{\mathrm{ij}}-\mathrm{x}^{\mathrm{cp}}{ }_{\mathrm{j}}\right) / \mathrm{s}_{\mathrm{j}}$. Элементы $\quad \mathrm{z}_{\mathrm{ij}}=\left(\mathrm{x}_{\mathrm{ij}}^{0}-\mathrm{x}^{\mathrm{cp}} \mathrm{j}_{\mathrm{j}}\right) \mathrm{s}_{\mathrm{j}} \quad$ матрицы стандартизованных отклонений не имеют размерности, и все ее столбцы имеют одинаковые дисперсии, равные единице. Это - одно из удобств для наших задач. Шаги при моделировании значений $\mathrm{Z}_{\mathrm{ij}}=\left(\mathrm{x}_{\mathrm{ij}}^{0} \mathrm{X}^{\mathrm{cp}} \mathrm{j}_{\mathrm{j}}\right) / \mathrm{s}_{\mathrm{j}} \mathrm{n} \mathrm{z}$-переменных отделены от шагов при вычислении выборочных средних $\mathrm{x}^{\mathrm{cp}}{ }_{\mathrm{j}}$ и дисперсий $\mathrm{s}_{\mathrm{j}}^{2}$ для реальных данных $\mathrm{x}_{\mathrm{ij}}^{0}$ $\mathrm{i}=1, \ldots, \mathrm{m}, \mathrm{j}=1, \ldots, n$. Векторы выборочных средних и дисперсий должны определяться из матрицы реальных данных $\mathrm{X}_{\mathrm{mn}}^{0}=\left\{\mathrm{x}_{\mathrm{ij}}\right\}$. Моделирование значений z-переменных проводится отдельно, a при преобразовании их в $\mathrm{x}^{0}-$ переменные $\mathrm{x}_{\mathrm{ij}}^{0}=\mathrm{z}_{\mathrm{ij}} \mathrm{s}_{\mathrm{j}}+\mathrm{x}^{\mathrm{cp}}{ }_{\mathrm{j}}, \quad \mathrm{i}=1, \ldots, \mathrm{m}, \quad \mathrm{j}=1, \ldots, \mathrm{n}, \quad$ можно использовать любые векторы выборочных средних и стандартных отклонений (дисперсий). При этом в полученной модельной матрице $\mathrm{Z}_{\mathrm{mn}}=\left[\mathrm{Z}_{1} \mid \mathrm{Z}_{2}\right], \mathrm{Z}_{2}=\mathrm{Z}_{1} \boldsymbol{\beta}$ можно переставлять местами строки - эти действия не влияют на значения элементов корреляционных матриц, вычисляемых ниже.

Такие матрицы $Z_{m n}=\left[Z_{1} \mid Z_{2}\right]$, будет иметь заданные целевые значения коэффициентов 


\begin{tabular}{|c|c|c|c|c|c|c|}
\hline \multirow{4}{*}{ Impact Factor: } & ISRA (India) & $=3.117$ & SIS (USA) & $=0.912$ & ICV (Poland) & $=6.630$ \\
\hline & ISI (Dubai, UAI & $=0.829$ & РИНЦ (Russia & $=0.156$ & PIF (India) & $=1.940$ \\
\hline & GIF (Australia) & $=0.564$ & ESJI (KZ) & $=8.716$ & IBI (India) & $=4.260$ \\
\hline & JIF & $=1.500$ & SJIF (Morocce & $=5.667$ & OAJI (USA) & $=0.350$ \\
\hline
\end{tabular}

регрессии $\beta_{1}, \ldots, \beta_{\mathrm{n}-1}$, таких что $\mathrm{Z}_{2}=\mathrm{Z}_{1} \boldsymbol{\beta}$. Тогда возможно проектирование рядов векторов значений коэффициентов регрессии $\boldsymbol{\beta}=\left(\beta_{1}, \ldots, \bigcap_{\mathrm{n}-1}\right)^{\mathrm{T}}$ и соответствующих им рядов матриц $\left\{\mathrm{Z}_{\mathrm{mn}}=\left[\mathrm{Z}_{1} \mid \mathrm{Z}_{2}\right]\right\}$ с заданными алгебраическими и когнитивными свойствами.

Суть нашего подхода может быть сведена не только к анализу выборочных коэффициентов корреляции и коэффициентов регрессии. А может и должна содержать другие извлекаемые знания, кроме знаний, интерпретируемых из равенства $\mathrm{Z}_{2}=\mathrm{Z}_{1} \boldsymbol{\beta}$. Здесь впервые рассматриваются элементы матрицы собственных векторов для моделирования, влияющих на вектор $\boldsymbol{\beta}=\mathrm{R}^{-1}{ }_{11} \mathrm{R}_{12}$ подматриц. Решаются 3 OC3: OC31, OC3 2 OC3 для ООМ МЛРА. Эти 3 задачи решаются, если в матрицу $\mathrm{C}_{11}$ собственных векторов математически введены - через индикаторы наличия знаний, значения весомых «весов».

Наши «обогащенные» знаниями коэффициенты регрессии $\beta_{1}, \ldots, \beta_{\mathrm{n}-1}$ имеют практически важные смысл и интерпретацию: «если банк увеличит на 1 тысячу тенге свои кредитные вложения, то банк потерпит убыток в 347,87 тенге, а если банк увеличит на 1 тысячу тенге свои вложения в ценные бумаги, то банк потерпит убыток в 225,42 тенге. т. е. банку в это время нельзя заниматься традиционными операциями» [1]. Теперь можно будет измерить степень неопределенности (риска) изменения zпеременной с весомым «весом».

Закон распределения значений 1-мерных zпеременных бывает неопределенным., что достигается применением обратной модели главных компонент (ОМ ГК) [2,3], для 1-мерных $\mathrm{z}$-переменных из R-, $\Lambda$-, $\boldsymbol{\beta}$-выборок не определены законы распределений, они модельно и гистограммно адекватны реальным многомерным таблицам «объект-свойства» [8-11].

\section{Модели и задачи}

Исходной гипотезой для рассматриваемой ниже является существование уравнения регрессии вида $\mathrm{z}_{\mathrm{n}}=\beta_{1} \mathrm{Z}_{1}+\beta_{2} \mathrm{Z}_{2}+\ldots+\beta_{\mathrm{n}-1} \mathrm{Z}_{\mathrm{n}-1}\left(\mathrm{Z}_{2}=\mathrm{Z}_{1} \boldsymbol{\beta}\right)$ Трудным местом ПЗ МЛРА $\mathrm{Z}_{\mathrm{mn}}=\left[\mathrm{Z}_{1} \mid \mathrm{Z}_{2}\right]=>\left(\mathrm{R}^{-1}{ }_{11}\right.$, $\left.\mathrm{R}_{12}, \boldsymbol{\beta}\right)$, является вычисление обратной матрицы для симметрической корреляционной матрицы «регрессоров» $\mathrm{R}_{11}$, которая может быть неполного ранга - тогда не существует для нее обратной матрицы. Если она «плохо обусловлена», то уменьшение числа обусловленности матрицы показывает насколько матрица близка к матрице неполного ранга (для квадратных матриц - к вырожденности). В работах [2-9] число обусловленности корреляционной матрицы измеряется значениями некоторых f-параметров из набора f-параметров $\left.\mathrm{f}_{1}\left(\Lambda_{(\mathrm{n}-1)(\mathrm{n}-1)}\right)=\lambda_{1}+\ldots+\lambda_{\mathrm{n}-1}\right)=$ $\mathrm{n}-1, \quad \mathrm{f}_{2}\left(\Lambda_{\mathrm{n}-1 \mathrm{n}-1}\right)=\left(\lambda^{2}{ }_{1}+\ldots+\lambda^{2}{ }_{\mathrm{n}-1}\right), \quad \mathrm{f}_{3}\left(\Lambda_{\mathrm{n}-1 \mathrm{n}-1}\right)=\lambda_{1} / \lambda_{\mathrm{n}-1}$, $\mathrm{f}_{4}\left(\Lambda_{(\mathrm{n}-1)(\mathrm{n}-1)}\right)=\left(\lambda_{1}+\ldots+\lambda_{\ell}\right) / \mathrm{n}-1<1, \quad \mathrm{f}_{5}\left(\Lambda_{(\mathrm{n}-1)(\mathrm{n}-1)}\right)=$ $\left.\lambda_{1} \times \lambda_{2} \times \lambda_{3} \times \ldots \times \lambda_{\mathrm{n}-1}, \quad \mathrm{f}_{6}\left(\Lambda_{(\mathrm{n}-1)(\mathrm{n}-1)}\right)=\lambda_{1} / \lambda_{2}+\ldots+\lambda_{\mathrm{n}-2} / \lambda_{\mathrm{n}-1}\right)$. Значение $\mathrm{f}$-параметра $\mathrm{f}_{3}\left(\Lambda_{(\mathrm{n}-1)(\mathrm{n}-1)}\right)=\lambda_{1} / \lambda_{\mathrm{n}-1} \quad$ 1 измеряет значение числа обусловленности, а остальные - близость (удаленность) от вырожденности корреляционной матрицы $\mathrm{R}_{\mathrm{nn}}$. Для нахождения значений $\mathrm{f}$-параметров необходимо решить прямую спектральную задачу (ПС3): $\mathrm{R}_{11}=>\left(\mathrm{C}_{11}, \Lambda_{11}\right), \quad$ где квадратная ортонормированная матрица $\mathrm{C}_{11}$ - матрица собственных векторов $\mathbf{c}_{\mathbf{j}}=\left(\mathrm{c}_{1 \mathrm{j}}, \mathrm{c}_{2 \mathrm{j}} \ldots \mathrm{c}_{\mathrm{n}-1, \mathrm{j}}\right)^{\mathrm{T}}, \mathrm{j}=1, \ldots, \mathrm{n}-1$. Они образуют ортогональную матрицу $\mathrm{C}_{11}=\left[\mathbf{c}_{1}\left|\mathbf{c}_{2}\right| \ldots \mid \mathbf{c}_{\mathbf{n}-1}\right], \quad$ согласованную с матрицей собственных чисел (со спектром) $\Lambda_{11}=\operatorname{diag}\left(\lambda_{1}, \lambda_{2}, \ldots, \lambda_{\mathrm{n}-1}\right), \quad \lambda_{1}>\ldots>\lambda_{\mathrm{n}-1}>0, \quad$ таким образом, что выполняются равенства $\mathrm{R}_{11} \mathrm{C}_{11}=\mathrm{C}_{11} \Lambda_{11}, \mathrm{C}^{\mathrm{T}}{ }_{11} \mathrm{C}_{11}=\mathrm{C}_{11} \mathrm{C}^{\mathrm{T}}{ }_{11}=\mathrm{I}_{(\mathrm{n}-1)(\mathrm{n}-1),} \quad$ где $\operatorname{diag}\left(\mathrm{R}_{11}\right)=(1, \ldots, 1), \operatorname{tr}\left(\mathrm{R}_{11}\right)=1+\ldots+1=\operatorname{tr}\left(\Lambda_{11}\right)=$ $\lambda_{1}+\ldots+\lambda_{n-1}=\mathrm{n}-1$. Матрицы $\mathrm{C}_{11}$ и $\Lambda_{11}$ вычисляются одновременно по известной корреляционной матрице $\mathrm{R}_{11}$. Матрица $\mathrm{R}_{11}$ вычисляется по стандартизованной выборке $Z_{\mathrm{m}(\mathrm{n}-1)}$ : $\mathrm{R}_{11}=(1 / \mathrm{m}) \mathrm{Z}^{\mathrm{T}}{ }_{1} \mathrm{Z}_{1}$. Элементы спектра $\Lambda_{11}=\operatorname{diag}\left(\lambda_{1}, \ldots, \lambda_{\mathrm{n}-1}\right), \quad \mathrm{n}>2, \quad$ являются [4] вышеприведенными измерителями как степени вырожденности, так и других свойств.

В работе [9] решена О3 МЛРА, а в статье [0] применена новая модель - ОМ МЛР. Ее решения $\left(\mathrm{R}^{(\ell)}{ }_{11}, \mathrm{R}^{(\ell)}{ }_{12}, \mathrm{Z}^{(\mathrm{t})}{ }_{1}, \quad \mathrm{Z}^{(\ell, \mathrm{t})}{ }_{2}\right), \quad$ где $\quad$ корреляционные матрицы $\mathrm{R}^{(\ell)}{ }_{11}$ моделируются в модели вида: $\left(\mathrm{n}, \varphi_{11}\right)=>\left(\mathrm{R}^{(\ell)}{ }_{11}\right)$, подматрицы $\mathrm{R}^{(\ell)}{ }_{12}$ вычисляются: $\mathrm{R}^{(\ell)}{ }_{12}=\mathrm{R}^{(\ell)}{ }_{11} \beta, \quad$ подматрицы $\mathrm{Z}^{(\ell, \mathrm{t})}{ }_{1} \quad$ являются решением О3 АГК: $\mathrm{R}^{(\ell)}{ }_{11} \Rightarrow\left(\mathrm{C}^{(\ell)}{ }_{11}, \Lambda^{(\ell)}{ }_{11}, \mathrm{Y}^{(\mathrm{t})} \mathrm{m}(\mathrm{n}-1)\right.$, $\left.\mathrm{Z}^{(\ell, \mathrm{t})} \mathrm{m}(\mathrm{n}-1)\right), \quad$ подматрица $\quad \mathrm{Z}^{(\ell, \mathrm{t})}$-решением Оптимизационной задачи №5, $\mathrm{t}=1, \ldots \mathrm{\kappa}_{\mathrm{t}}<\infty$, $\ell=1, \ldots, \mathrm{k}_{\ell}<\infty$.

Выборки $Z^{(t)}{ }_{1} Z^{(\ell, t)}$ OM ГК удовлетворяют соотношениям: $\quad(1 \backslash \mathrm{m}) \mathrm{Z}^{(\mathrm{t}) \mathrm{T}}{ }_{1} \mathrm{Z}^{(\mathrm{t})}{ }_{1}=\mathrm{R}^{(\ell)}{ }_{11},(1 \backslash \mathrm{m}) \mathrm{Z}^{(\mathrm{t}) \mathrm{T}}{ }_{1}$ $\mathrm{Z}^{(\ell, \mathrm{t})}{ }_{2}=\mathrm{R}^{(\ell)}{ }_{12}, \quad(1 \backslash \mathrm{m}) \mathrm{Z}^{(\ell, \mathrm{t}) \mathrm{T}}{ }_{2} \mathrm{Z}^{(\ell, \mathrm{t})}{ }_{2}=\mathrm{R}_{22}=1 . \quad$ Матрицы $\left.\mathrm{C}^{(\ell)}{ }_{11}, \Lambda^{(\ell)}{ }_{11}, \mathrm{Y}^{(\mathrm{t})} \mathrm{m}(\mathrm{n}-1), \mathrm{Z}^{(\ell, \mathrm{t})}{ }_{\mathrm{m}(\mathrm{n}-1)}\right)$ из решаемых задач используются для достижения требуемых равенств, а также удовлетворяют соотношениям ОМ ГК, доказанным в Теореме о $\Lambda$-выборках [9].

\section{Обратная обобщенная модель множественной линейной регрессии}

Необходимо демонстрирующие всевозможные динамики рассматриваемых нами агрегированных показателей. Динамики этих показателей покажут оптимистические или неблагоприятные тенденции в периоды времени, наличие которых мы будем определять по значениям показателей, по коэффициентам корреляции, по значениям 


\begin{tabular}{|c|c|c|c|c|c|c|}
\hline \multirow{4}{*}{ Impact Factor: } & ISRA (India) & $=3.117$ & SIS (USA) & $=0.912$ & ICV (Poland) & $=6.630$ \\
\hline & ISI (Dubai, UAI & $=0.829$ & РИНЦ (Russia & $=0.156$ & PIF (India) & $=1.940$ \\
\hline & GIF (Australia) & $=0.564$ & ESJI (KZ) & $=8.716$ & IBI (India) & $=4.260$ \\
\hline & JIF & $=1.500$ & SJIF (Morocce & $=5.667$ & OAJI (USA) & $=0.350$ \\
\hline
\end{tabular}

коэффициентов регрессии $\beta_{1}, \ldots, \quad \beta_{\mathrm{n}-1}, \quad$ по коэффициентам эластичности переменной $\mathrm{z}_{\mathrm{n}}$ по объясняющей переменной $\mathrm{z}_{\mathrm{j}}$ с номером $\mathrm{j}$, где $\mathrm{j}$ может принимать одно из значений $1,2 \ldots, n-1$.

В данной работе управляемым (входным) параметром модели, генерирующей модельные данные, являются значения коэффициентов регрессии $\beta_{1}, \ldots, \beta_{\mathrm{n}-1}$ при переменных $\mathrm{Z}_{1}, \mathrm{Z}_{2}, \ldots, \mathrm{Z}_{\mathrm{n}-1}$ в уравнении регрессии. $\mathrm{B}$ общеизвестной ПМ МЛРА $\mathrm{Z}_{\mathrm{mn}}=\left[\mathrm{Z}_{1} \mid \mathrm{Z}_{2}\right]=>\left(\mathrm{R}^{-1}{ }_{11}, \mathrm{R}_{12}, \beta\right) \quad$ входным объектом является реальная стандартизованная многомерная выборка $Z_{m n}=\left[Z_{1} \mid Z_{2}\right]$, а выходнымвектор коэффициентов регрессии $\beta=\left(\beta_{1}, \ldots, \beta_{\mathrm{n}-1}\right)$. В нашей новой ООМ МЛРА входные и выходные объекты меняются местами: входным объектом является вектор $\beta=\left(\beta_{1}, \ldots, \beta_{\mathrm{n}-1}\right)$ и пара матриц $\left(\mathrm{C}^{+}{ }_{11}, \Lambda^{+}{ }_{11}\right)$, а входными объектами $\left.\mathrm{R}^{+}{ }_{11}, \mathrm{R}^{+}{ }_{12}, \mathrm{Z}^{(\mathrm{t})}{ }_{1} \mid \mathrm{Z}^{(\mathrm{t})}{ }_{2}\right], \mathrm{t}=1, \ldots, \mathrm{K}_{\mathrm{t}}<\infty$.

Как показано ниже для единственного вектора коэффициентов регрессии $\beta=\left(\beta_{1}, \ldots, \beta_{\mathrm{n}-1}\right)$ существует бесконечное множество стандартизованных многомерных выборок $\mathrm{Z}_{\mathrm{mn}}=\left[\mathrm{Z}_{1} \mid \mathrm{Z}_{2}\right]$, являющиеся многомерными $\Lambda_{11^{-}}$ выборками с свойствами, доказанными в теореме о $\Lambda$-выборках [9]. Обратная обобщенная модель множественной линейной регрессии (ООМ МЛРА) имеет схематическое изображение вида: $\beta=>\left[\mathrm{R}^{(\ell)}{ }_{11}, \mathrm{R}^{(\ell)}{ }_{12} \mathrm{Z}^{(\ell, \mathrm{t})}{ }_{1} \mid \mathrm{Z}^{(\ell, \mathrm{t})}{ }_{2}\right], \quad \mathrm{t}=1, \ldots, \mathrm{K}_{\mathrm{t}}<\infty$, $\ell=1, \ldots, \mathrm{K}_{\ell}<\infty$.

\section{Обратная множественного обобщенная задача анализа}

Пусть нам даны перечень наименований и число n-1 независимых переменных, т.е. задано число «регрессоров» $\mathrm{Z}_{\mathrm{i}}, \ldots, \mathrm{Z}_{\mathrm{i}, \mathrm{n}-1}$ и заданы значения коэффициентов регрессии $\quad \beta_{1}, \ldots, \beta_{\mathrm{n}-1}$, функционально определяющих значения зависимой переменной $\mathrm{z}_{\mathrm{n}}: \mathrm{z}_{\mathrm{n}}=\mathbf{z} \beta, \mathbf{z}=\left(\mathrm{z}_{\mathrm{i} 1}, \ldots, \mathrm{z}_{\mathrm{i}, \mathrm{n}-1}\right)$, $\boldsymbol{\beta}=\mathrm{R}^{-1}{ }_{11} \mathrm{R}_{12}$.

Требуется моделировать значения выборочных коэффициентов корреляции между n-1 независимыми z-переменными из подматрицы $\mathrm{R}_{11}$ коэффициентов корреляций только независимых переменных («регрессоров»). Требуется моделировать значения выборочных коэффициентов корреляции $\mathrm{r}_{1 \mathrm{n}}, \ldots, \mathrm{r}_{\mathrm{n}-1, \mathrm{n}}$ между зависимой $\mathrm{z}$-переменной $\mathrm{z}_{\mathrm{n}}$ и независимыми $\mathrm{z}$ переменными $\mathrm{z}_{1}, \ldots, \mathrm{z}_{\mathrm{n}-1}$, объединенных в векторстолбец $\mathrm{R}_{12}=\left(\mathrm{r}_{1 \mathrm{n}}, \ldots, \mathrm{r}_{\mathrm{n}-1, \mathrm{n}}\right)^{\mathrm{T}}$.

Требуется моделировать многомерную $\mathrm{m} * \mathrm{n}$ выборку $\mathrm{Z}_{\mathrm{mn}}=\left[\mathrm{Z}_{1} \mid \mathrm{Z}_{2}\right]$ значений $\mathrm{n}$ Z-переменных, удовлетворяющих равенствам из ПМ МЛРА $\mathrm{Z}_{\mathrm{mn}}=\left[\mathrm{Z}_{1} \mid \mathrm{Z}_{2}\right]=>\left(\mathrm{R}^{-1}{ }_{11}, \mathrm{R}_{12}, \beta\right):(1 \backslash \mathrm{m}) \mathrm{Z}_{1}^{\mathrm{T}} \mathrm{Z}_{1}=\mathrm{R}_{11}$,

$\mathrm{Z}_{\mathrm{mn}}=\left[\mathrm{Z}_{1} \mid \mathrm{Z}_{2}\right]=\left\{\left(\mathrm{Z}_{\mathrm{i} 1}, \ldots, \mathrm{Z}_{\mathrm{i}, \mathrm{n}-1} \mid \mathrm{Z}_{\mathrm{in}}\right)\right\}, \quad(1 \backslash \mathrm{m}) \mathrm{Z}_{1}^{\mathrm{T}} \mathrm{Z}_{1}=\mathrm{R}_{11}$, $(1 \backslash \mathrm{m}) \mathrm{Z}_{1}^{\mathrm{T}} \mathrm{Z}_{2}=\mathrm{R}_{12}, \quad \mathrm{Z}_{\mathrm{n}}=\mathbf{z R}^{-1}{ }_{11} \mathrm{R}_{12}=\mathbf{z} \beta, \quad \mathbf{z}=\left(\mathrm{Z}_{1}, \ldots, \mathrm{Z}_{\mathrm{n}-1}\right)$,
$\boldsymbol{\beta}=\mathrm{R}^{-1}{ }_{11} \mathrm{R}_{12}$. При этом матрица $\mathrm{R}_{11} \mathrm{n}-1$ независимых $\mathrm{Z}$-переменных имеет заданные матрицы собственных векторов $\mathrm{C}_{11}$, собственных чисел $\Lambda_{11}$ : $\mathrm{R}_{11} \mathrm{C}_{11}=\mathrm{C}_{11} \Lambda_{11} \mathrm{R}^{-1}{ }_{11} \mathrm{R}_{12}=\mathrm{C}_{11} \Lambda^{-1}{ }_{11} \mathrm{C}^{\mathrm{T}}{ }_{11} \mathrm{R}_{12}$, где матрицы $\mathrm{R}_{11}, \mathrm{R}_{12}, \mathrm{R}^{\mathrm{T}}{ }_{12}$ образуют (n-1) $\times(\mathrm{n}-1)-,(\mathrm{n}-1)$ $\times 1-, 1 \times(n-1)$-разбиения корреляционной матрицы $\mathrm{R}_{\mathrm{nn}}$. Матрица $\mathrm{C}_{11}$ преобразуется в матрицу собственных векторов $\mathrm{C}^{+}{ }_{11}, \quad$ обязательно содержащую заданное множество индикаторов присутствия знаний. Извлечение знаний должно проводиться по когнитивной модели, основанной на математической модели ХотеллингаЖанатауова.

Входным объектом обратной задачи множественной линейной регрессии (О3 МЛРА) является вектор $\beta_{\mathrm{R}}=\left(\beta_{1}, \ldots, \beta_{\mathrm{n}-1}\right)$, а в решаемой здесь Обобщенной О3 МЛРА (ООЗ МЛРА) вторым входным объектом является матрица собственных векторов $\mathrm{C}^{+}{ }_{11}$ с весомыми по абсолютной величине компонентами $c_{\mathrm{kj}}$, таких что $\left|\mathrm{c}_{\mathrm{kj}}\right| \geq \mathrm{c}_{\mathrm{j}}$, $\mathrm{j}=1, \ldots, \mathrm{n}-1$, из $\mathrm{n}$ компонент j-го собственного вектора $\mathbf{c}_{\mathbf{j}}=\left(\mathrm{c}_{1 \mathrm{j}}, \mathrm{c}_{2 \mathrm{j}} \ldots \mathrm{c}_{\mathrm{nj}}\right)^{\mathrm{T}}, \mathrm{j}=\in\{1, \ldots, \mathrm{n}\}$. Критерий «весомости»-пороговое значение $\mathrm{c}_{\mathrm{j}}$, - задается в соответствии с градациями в шкале Чэддока. Число интервалов градаций равно 5, разных значений $\mathrm{c}_{\mathrm{j}+1} \neq \mathrm{c}_{\mathrm{j}}$ может быть 5 или пороговое значение $\mathrm{c}_{\mathrm{j}}$ может быть одинаковым для всех j.

Решение подзадачи 1 состоит из 2 шагов. Шаг 1:(n, $\left.\varphi_{11}\right)=>\left(\Lambda_{11}\right)$. Шаг 1: $)=>\left(\Lambda_{11}=>\left(\mathrm{C}_{11}, \Lambda_{11}\right)\right.$.

Далее фиксируем начальные матрицы собственных векторов $\mathrm{C}_{11}$, собственных чисел $\Lambda_{11}$ и решаем задачу формирования индикаторов присутствия знаний (индикаторные элементы) в матрице $\mathrm{C}_{11}$. $\mathrm{B}$ результате решения задачи формирования индикаторов присутствия знаний и решения будут смоделированы 2 матрицы $\mathrm{C}^{+}{ }_{11}, \Lambda^{+}{ }_{11}$.

Далее моделируем $\left(\mathrm{C}^{+}{ }_{11}, \Lambda^{+}{ }_{11}\right)$-выборку $\mathrm{Z}^{(\mathrm{t})}{ }_{1}$ значений $\mathrm{Z}$-переменных $\left(\mathrm{z}_{1}, \ldots \mathrm{z}_{\mathrm{n}-1}\right)$, образующих подматрицу $Z^{(\mathrm{t})} \mathrm{m}(\mathrm{n}-1) \quad$ матрицы $\quad Z^{(\mathrm{t})}{ }_{\mathrm{mn}}=\left[Z^{(\mathrm{t})} \mid Z^{(\mathrm{t})}{ }_{2}\right]$. Этим мы реализуем схему варианта ОМ ГК: $\left(\mathrm{C}^{+}{ }_{11}, \Lambda^{+}{ }_{11}\right)=>\left(\mathrm{R}^{+}{ }_{11}, \Lambda_{11}, \mathrm{Y}^{(\mathrm{t})}{ }_{\mathrm{m}(\mathrm{n}-1)}, \mathrm{Z}^{(\mathrm{t})}{ }_{\mathrm{m}(\mathrm{n}-1)}\right), \mathrm{t}=1, \ldots, \mathrm{K}_{\mathrm{t}}<\infty$. Но рам нужно в дополнение смоделировать значения $\mathrm{Z}$-переменной $\mathrm{Z}_{\text {in }}$ из подматрицы $\mathrm{Z}^{(\mathrm{t})}{ }_{2}$ матрицы $\mathrm{Z}_{\mathrm{mn}}=\left[\mathrm{Z}_{1} \mid \mathrm{Z}_{2}\right]=\left\{\left(\mathrm{Z}_{\mathrm{i} 1}, \ldots, \mathrm{Z}_{\mathrm{i}, \mathrm{n}-1} \mid \mathrm{Z}_{\mathrm{in}}\right)\right\}$. Тогда матрица $\mathrm{R}^{+}{ }_{\mathrm{nn}}$ будет такой, что будут выполняться соотношения $\quad \beta_{\mathrm{R}}=\mathrm{R}\left({ }^{+}\right)-1{ }_{11} \mathrm{R}^{+}{ }_{12}, \quad(1 \backslash \mathrm{m}) \mathrm{Z}_{1}^{\mathrm{T}} \mathrm{Z}_{1}=\mathrm{R}^{+}{ }_{11}$, $(1 \backslash \mathrm{m}) \mathrm{Z}_{1}^{\mathrm{T}} \mathrm{Z}_{2}=\mathrm{R}^{+}{ }_{12}$. Здесь матрица $\mathrm{R}^{+}{ }_{11}$ определена по формуле, а подматрица $\mathrm{R}^{+}{ }_{12}$. и подматрица 2 неизвестны. Значения их элементов будут смоделированы ниже после решения Оптимизационной Задачи.

Выходным объектом ООЗ МЛРА, т.е. решением, является многомерная выборка $\mathrm{Z}_{\mathrm{mn}}=\left[\mathrm{Z}_{1} \mid \mathrm{Z}_{2}\right]=\left\{\left(\mathrm{Z}_{\mathrm{i} 1}, \ldots, \mathrm{Z}_{\mathrm{i}, \mathrm{n}-1} \mid \mathrm{Z}_{\mathrm{in}}\right)\right\}$, матрица $\mathrm{R}_{\mathrm{nn}}$ такая, что выполняются соотношения $\beta_{\mathrm{R}}=\mathrm{R}^{-1}{ }_{11} \mathrm{R}_{12}$, $(1 \backslash \mathrm{m}) \mathrm{Z}_{1}^{\mathrm{T}} \mathrm{Z}_{1}=\mathrm{R}_{11},(1 \backslash \mathrm{m}) \mathrm{Z}_{1}^{\mathrm{T}} \mathrm{Z}_{2}=\mathrm{R}_{12}$. В соответствии с 


\begin{tabular}{llllll} 
& ISRA (India) $=\mathbf{3 . 1 1 7}$ & SIS (USA) $=\mathbf{0 . 9 1 2}$ & ICV (Poland) & $\mathbf{= 6 . 6 3 0}$ \\
Impact Factor: & ISI (Dubai, UAE) $=\mathbf{0 . 8 2 9}$ & PUHЦ (Russia) $=\mathbf{0 . 1 5 6}$ & PIF (India) & $=\mathbf{1 . 9 4 0}$ \\
& GIF (Australia) $=\mathbf{0 . 5 6 4}$ & ESJI (KZ) & $\mathbf{8 . 7 1 6}$ & IBI (India) & $\mathbf{4 . 2 6 0}$ \\
& JIF & $\mathbf{1 . 5 0 0}$ & SJIF (Morocco) $=\mathbf{5 . 6 6 7}$ & OAJI (USA) & $\mathbf{= 0 . 3 5 0}$ \\
\hline
\end{tabular}

вертикальным разбиением матрицы $Z_{\mathrm{mn}}=\left[\mathrm{Z}_{1} \mid \mathrm{Z}_{2}\right]$, где $Z_{1}-$ m-на-(n-1) матрица, $Z_{2}-$ m-на-1 матрица, матрица $R_{n n}$ разбита на 4 блока: подматрицы $\mathrm{R}_{11}, \mathrm{R}_{12} \quad \mathrm{R}_{21}=\mathrm{R}^{\mathrm{T}}{ }_{12}$, и элемент $\mathrm{r}_{\mathrm{nn}}=1$.

Схематически данную О3 МЛРА изобразим пока так: $\left(\mathrm{m}, \mathrm{n}, \varphi_{11}, \boldsymbol{\beta}\right)=>\left(\mathrm{R}_{11}, \mathrm{R}_{12}, \mathrm{Z}_{1}, \mathrm{Z}_{2}\right) . \mathrm{B}$ процессе ее решения схема будет конкретизироваться.

Подзадачу 1 для ОЗ МЛРА обозначим так: $\left(\mathrm{n}, \varphi_{11}\right)=>\left(\mathrm{R}_{11}\right)$. Так как $\mathrm{R}_{11} \mathrm{C}_{11}=\mathrm{C}_{11} \Lambda_{11}$, то имеем $\mathrm{R}_{11}=\mathrm{C}_{11} \Lambda_{11} \mathrm{C}^{\mathrm{T}}{ }_{11}$. Располагая двумя входными объектами-числами (n, $\left.\varphi_{11}\right)$ можно смоделировать бесконечно много спектров $\Lambda_{11}$. Для моделирования спектра $\Lambda_{11}=\Lambda_{\mathrm{n}-1)(\mathrm{n}-1)}=\operatorname{diag}\left(\lambda_{1}, \ldots\right.$, $\left.\lambda_{\text {n-1 }}\right)$, элементы которого по регулируемым величинам f-параметров должны удовлетворять нас и соответствовать выбранному значению $\varphi_{11}$, мы воспользуемся математической моделью спектра неизвестной корреляционной матрицы [ ]. Имея спектр $\Lambda_{11}$ мы далее решаем ОС3 1: $\Lambda_{11}=>\left(\mathrm{R}^{(\ell)}{ }_{11}, \mathrm{C}^{(\ell)}{ }_{11}\right), \ell=1, \ldots, \mathrm{k}_{\ell}=1000<\infty$.

Выберем одну пару матриц $\left(\Lambda_{11}, C^{(\ell)}{ }_{11}\right)$. Эта матрица $\mathrm{C}^{(\ell)}{ }_{11} \quad$ (Таблица 1) определяет корреляционную матрицу $\mathrm{R}_{11}=\mathrm{C}^{(\ell)}{ }_{11} \Lambda_{11} \mathrm{C}^{(\ell) \mathrm{T}}{ }_{11}$, нас не удовлетворяющую, так как в элементы матрицы $\mathrm{C}^{(\ell)}{ }_{11}$ мы не внедрили желаемые индикаторы присутствия знаний (индикаторные элементы). Иначе говоря, не «обогатили» матрицу $\mathrm{C}^{(\ell)} 11$ знаниями, выявляемые при когнитивном моделировании [3-6]. Назначим значения элементов этих матриц начальными значениями в Оптимизационной Задаче - важной задаче в ООМ МЛРА. Откроем надстройку «Поиск решения» в ЭТ Excel, поставим курсор компьютерной мыши (computer mouse) на целевую ячейку с адресной формулой $\lambda_{1}, \ldots, \lambda_{\mathrm{n}-1}=\mathrm{n}-1$ и нажмем на кнопку "Выполнить". Происходит запуск процедуры Solver. В результате работы процедуры Solver имеем новую желаемую матрицу $\mathrm{C}^{+}{ }_{11}$, удовлетворяющую всем условиям из формулировки ООЗ МЛРА.

Для внедрения индикаторов решаем Оптимизационную Задачу, в результате формируются индикаторы присутствия знаний, они видны среди элементов матрицы $\mathrm{C}^{+}{ }_{11}$, (Таблица 3). Решениями Оптимизационной Задачи являются 2 матрицы $\mathrm{C}^{+}{ }_{11}, \Lambda^{+}{ }_{11}$, но используем только одну матрицу $\mathrm{C}^{+}{ }_{11}$. Она матрица $\mathrm{C}^{+}{ }_{11}$, содержит индикаторы присутствия знаний, которые были внедрены в нее при решении Оптимизационной Задачи.

Решаемая ниже обратная спектральная задача (назовем ее ОСЗ для ООМ МЛРА) является другой обрат из ной спектральной задачей, отличной $\quad$ от OC3 1 [13]: $\Lambda_{11}=>\left(\mathrm{C}^{(\ell)}{ }_{11}, \mathrm{R}^{(\ell)}{ }_{11}\right)$, $\ell=1, \ldots, \mathrm{k}_{\ell}<\propto$, и от ПС3: $\mathrm{R}_{11}=>\left(\mathrm{C}_{11}, \Lambda_{11}\right)$. Схематично обозначим ОСЗ для ООМ МЛРА так:( $\mathrm{C}_{11}$, $\left.\Lambda_{11}\right)=>\left(\mathrm{C}^{+}{ }_{11}, \Lambda^{+}{ }_{11}, \mathrm{R}^{+}{ }_{11}\right)$. Наша ОСЗ для ООМ МЛРА не решается путем модификации алгоритма решения ОСЗ 1 из [13]. ОСЗ для ООМ МЛРА является новой задачей и являлся недостающим звеном в спектральных задачах с симметрической матрицей. Наша ООЗ МЛРА является обратной к ПС3: $\mathrm{R}_{(\mathrm{n}-1)(\mathrm{n}-1)}=>\left(\mathrm{C}_{(\mathrm{n}-1)(\mathrm{n}-1)}, \Lambda_{(\mathrm{n}-1)(\mathrm{n}-1)}\right)$ в ином аспекте в матрице $\mathrm{C}^{+}{ }_{11}$ содержатся математические индикаторы наличия желаемых знаний. Этого нет в Прямой Задаче диагонализации известной выборочной корреляционной матрицы $\mathrm{R}_{11}$, решаемой для известной симметрической матрицы $\mathrm{R}_{11}=\mathrm{R}^{\mathrm{T}}{ }_{11}$.

Преимущества модельных матрицы $\mathrm{C}^{+} 11$ собственных векторов и $\left(\beta, \mathrm{C}^{+} 11\right)$-выборок ООМ МЛРА

В статье [7] сформулирована и решена новая Обратная Спектральная Задача №2 (ОС3 для ООМ МЛРА при размерности n-1). В статье [7] рассматривалась ОС32 для корреляционной матрицы $\mathrm{R}_{\mathrm{nn}}$, имеющей полный ранг, равный n. B нашей новой задаче корреляционная матрица $\mathrm{R}_{\mathrm{nn}}$ имеет неполный ранг. Ее ранг равен $\mathrm{n}-1$, ибо линейная связь вида $\mathrm{Z}_{\mathrm{n}}=\mathbf{z} \beta$ уменьшает ранг матрицы $R_{n n}=(1 \backslash \mathrm{m}) \mathrm{Z}^{\mathrm{T}}{ }_{\mathrm{mn}} \mathrm{Z}_{\mathrm{mn}}$ на 1 .

В отличие от статьи [7] здесь мы рассматриваем ОС 32 для моделирования матрицы собственных векторов $\mathrm{C}^{+}{ }_{11}$ с неизвестной подматрицей $\mathrm{R}^{+}{ }_{11}$ неизвестной полной корреляционной матрицы $\mathrm{R}^{+}$. Если к подматрице $\mathrm{R}^{+}{ }_{11}$ добавить подматрицы $\mathrm{R}^{+}{ }_{12}, \mathrm{R}^{+\mathrm{T}}{ }_{12}, \mathrm{R}^{+}{ }_{22}=(1)$, то получили бы матрицу $\mathrm{R}^{+}$n. Но подматрицы $\mathrm{R}_{12}$, $\mathrm{R}^{\mathrm{T}}{ }_{12}, \mathrm{R}_{22}=(1)$ всегда определены, если известна подматрица $\mathrm{R}_{11}$. Матрица $\mathrm{R}^{+}$n восстановима, ибо подматрица $\mathrm{R}^{+}{ }_{22}=(1)$ имеет размерность 1 -на-1 и имеет единственный элемент, равный 1 , а по условию задачи подматрица $\mathrm{R}^{+} 12$ вычисляется через подматриц $\mathrm{R}^{+} 11$, так как имеем равенство $\mathrm{R}^{+}{ }_{11} \boldsymbol{\beta}=\mathrm{R}^{+}{ }_{12}$.

Наличие известных значений элементов вектора $\boldsymbol{\beta}$ и известных значений элементов матрицы $\mathrm{R}^{+} 11$, имеющей свою матрицу $\mathrm{C}^{+}{ }_{11} \mathrm{c}$ заданными пользователем по тем или иным причинам индикаторами присутствия «значимых» знаний, которые были «внедрены» в нее перед решением Оптимизационной Задачи для ООМ МЛРА, является явным преимуществом. Этими свойствами ООМ МЛРА желательно наделить и другие многомерные модели ПМСА.

Решение Оптимизационной Задачи обеспечивает нам нахождение искомых 2 подматриц $\mathrm{Z}_{1}, \mathrm{Z}_{2}$ таких, что $\mathrm{Z}_{2}=\mathrm{Z}_{1} \boldsymbol{\beta}$.

Но мы должны вложить в начальную матрицу $\mathrm{C}_{11}=\left[\mathbf{c}_{1}\left|\mathbf{c}_{2}\right| \ldots \mid \mathbf{c}_{\mathrm{n}-1}\right]$ «цифровые знания», применяя решение ОСЗ 2 [14]. Полученную после решения ОС32 для ООМ МЛРА «обогащенную» 


\begin{tabular}{|c|c|c|c|c|c|c|}
\hline \multirow{4}{*}{ Impact Factor: } & ISRA (India) & $=3.117$ & SIS (USA) & $=0.912$ & ICV (Poland) & $=6.630$ \\
\hline & ISI (Dubai, UAI & $=0.829$ & РИНЦ (Russia & $=0.156$ & PIF (India) & $=1.940$ \\
\hline & GIF (Australia) & $=0.564$ & ESJI (KZ) & $=8.716$ & IBI (India) & $=4.260$ \\
\hline & JIF & $=1.500$ & SJIF (Morocce & $=5.667$ & OAJI (USA) & $=0.350$ \\
\hline
\end{tabular}

матрицу $\mathrm{C}^{+} 11$ применяем и моделируем соответствующие 2 подматрицы $\mathrm{Z}^{+}, \mathrm{Z}^{+}{ }_{2}$ такие, что $\mathrm{Z}^{+}{ }_{2}=\mathrm{Z}^{+}{ }_{1} \boldsymbol{\beta}$. Тогда модельная выборка $\mathrm{Z}^{+}{ }_{\mathrm{mn}}=\left[\mathrm{Z}^{+}{ }_{1} \mid \mathrm{Z}^{+}{ }_{2}\right]$ будучи подвергнута процессу извлечения из нее знаний с применением математической модели главных компонент [4-6], с применением когнитивного моделирования для $\mathrm{y}$ - и $\mathrm{z}$ переменных [4-6], с корректным решением смысловых задач [15], позволит извлечь из матрицы $\mathrm{C}^{+}{ }_{11}$ «цифровые знания». Описание обратного процесса извлечения введенных знаний проводится с применением когнитивного моделирования будет опубликовано в другой статье.

Наша модельная выборка $\mathrm{Z}^{+}{ }_{\mathrm{mn}}=\left[\mathrm{Z}^{+} \mid \mathrm{Z}_{2}^{+}\right]$ адекватна (модельно и гистограммно [7-9]) и мы сможем как моделировать, так и извлекать «цифровые знания» в плохо формализуемых событиях, ситуациях [7-9]. Заметим, что извлекать «цифровые знания» мы можем только в рамках математической модели главных компонент [4-6], математической модели МЛРА [1-3].

\section{МЛРА}

Обратная Спектральная Задача для ООМ

Пусть верна гипотеза: $\mathrm{n}$ z-переменных условно разделены на 2 части первые n-1 zпеременных содержат существенные знания и достаточно сильно влияют на одну z-переменную $z_{n}$ согласно линейной модели вида $\mathrm{z}_{\mathrm{n}}=\beta_{1} \mathrm{z}_{1}+\beta_{2} \mathrm{z}_{2}+\ldots+\beta_{\mathrm{n}-1} \mathrm{z}_{\mathrm{n}-1}$, где известны значения регрессионных коэффициентов $\beta_{1}, \ldots, \beta_{\mathrm{n}-1}$.

В векторном виде эта линейная модель имеет вид $Z_{2}=Z_{1} \boldsymbol{\beta}$, где вектор регрессионных коэффициентов $\boldsymbol{\beta}=\left(\bigcap_{1}, \ldots, \beta_{\mathrm{n}-1}\right)^{\mathrm{T}}$, m значений всех $\mathrm{n}$ $\mathrm{Z}$-переменных образует 2 подматрицы $\mathrm{Z}_{1}, \mathrm{Z}_{2}$ размерностей m-на-(n-1) и m-на-1. Объединение $\mathrm{m}$ значений всех z-переменных в одно множество из $\mathrm{m}$ значений $\mathrm{n} z$-переменных имеет форму m-на$\mathrm{n}$ матрицы $\left.\mathrm{Z}_{\mathrm{mn}}=\left[\mathrm{Z}_{1}\right\rfloor \mathrm{Z}_{2}\right]$. В 1 МЛРА заданы значения регрессионных коэффициентов $\beta_{1}, \ldots$, $\beta_{\mathrm{n}-1}$.

Требуется найти модельные т значений всех n Z-переменных образующих 2 подматрицы $\mathrm{Z}_{1}, \mathrm{Z}_{2}$ для m-на-n матрицы $Z_{\mathrm{mn}}$. При условии, что вектор B, удовлетворяет стандартному условию $\boldsymbol{\beta}=\mathrm{R}^{-1}{ }_{11} \mathrm{R}_{12}$. Здесь, как известно, подматрицы $\mathrm{R}_{11}, \mathrm{R}_{12}, \mathrm{R}_{12}^{\mathrm{T}}, \mathrm{R}_{22}$ определяются по формулам $(1 \backslash \mathrm{m}) \mathrm{Z}_{1}^{\mathrm{T}} \mathrm{Z}_{1}=\mathrm{R}_{11}, \quad(1 \backslash \mathrm{m}) \mathrm{Z}_{1}^{\mathrm{T}} \mathrm{Z}_{2}=\mathrm{R}_{12}, \quad(1 \backslash \mathrm{m}) \mathrm{Z}_{2}^{\mathrm{T}} \mathrm{Z}_{2}=$ $\mathrm{R}_{22}=(1)$ и являются разбиениями корреляционной матрицы $\mathrm{R}_{\mathrm{nn}}=(1 \backslash \mathrm{m}) \mathrm{Z}_{\mathrm{mn}}^{\mathrm{T}} \mathrm{Z}_{\mathrm{mn}}$, соответствующих разбиению $Z_{\mathrm{mn}}=\left[\mathrm{Z}_{1} \mid \mathrm{Z}_{2}\right]=\left\{\left(\mathrm{Z}_{\mathrm{i}}, \ldots, \mathrm{Z}_{\mathrm{i}, \mathrm{n}-1} \mid \mathrm{Z}_{\mathrm{in}}\right)\right\}$.

\footnotetext{
Формулировка ОЗ МЛРА для матрицы собственных векторов $\mathrm{C}^{+}{ }_{11}$
}

Модельная подматрица $\mathrm{R}^{+}{ }_{11}$ не известна и должна иметь заданную матрицу собственных векторов $\mathrm{C}^{+}{ }_{11}$. В начальную матрицу $\mathrm{C}_{11}$ мы должны ввести неизменяемые весомые значения $\mathrm{c}^{+}{ }_{\mathrm{kj}}$ такие, что они по абсолютной величине «весомы»: $\left|\mathrm{c}^{+}{ }_{\mathrm{kj}}\right|>\mathrm{c}_{\mathrm{j}}$. Они являются выделенными компонентами [14] собственных векторов $\left[\mathbf{c}_{1}|\ldots| \mathbf{c}_{\mathbf{n}-1}\right]$. Они могут быть произвольными, но удовлетворяющими соотношениям $\left|\mathrm{c}^{+}{ }_{\mathrm{kj}}\right|>\mathrm{c}_{\mathrm{j}}$. Должна быть задана начальная пара матриц $\left(\mathrm{C}_{11}, \Lambda_{11}\right)$ собственных векторов $\mathrm{C}_{11}$ и собственных чисел $\quad \Lambda_{11}=\operatorname{diag}\left(\lambda_{1}, \ldots, \lambda_{\mathrm{n}-1}\right), \quad \lambda_{1}+\ldots+\lambda_{\mathrm{n}-1}=\mathrm{n}-1$, $\lambda_{1} \geq \ldots \geq \lambda_{\mathrm{n}-1}$. Наличие этой пары обеспечивается решением ОС3 1 [13]: $\Lambda_{11}=>\left(\mathrm{C}_{11}, \mathrm{R}_{11}\right)$. Здесь мы не указываем номер $\ell=1, \ldots, \mathrm{k}_{\ell}<\propto$. Bce $\mathrm{n}-1$ собственные векторы $\mathbf{c}_{\mathrm{j}}=\left(\mathrm{c}_{1 \mathrm{j}}, \mathrm{c}_{2 \mathrm{j}} \ldots \mathrm{c}_{\mathrm{n}-1, \mathrm{j}}\right)^{\mathrm{T}}$, расположенные по n-1 столбцам матрицы $\mathrm{C}_{11}=\left[\mathbf{c}_{1}\left|\mathbf{c}_{2}\right| \ldots \mid \mathbf{c}_{\mathrm{n}-1}\right]$, согласованы со своим спектром $\Lambda_{11}=\operatorname{diag}\left(\lambda_{1}, \ldots \lambda_{\text {n-1 }}\right) \quad$ таким образом, что выполняются равенства: $\mathrm{R}_{11}=\mathrm{C}_{11} \Lambda_{11} \mathrm{C}^{\mathrm{T}}{ }_{11}$, $\mathrm{R}_{11} \mathrm{C}_{11}=\mathrm{C}_{11} \Lambda_{11}, \quad \mathrm{C}^{\mathrm{T}}{ }_{11} \mathrm{C}_{11}=\mathrm{C}_{11} \mathrm{C}^{\mathrm{T}}{ }_{11}=\mathrm{I}_{\mathrm{nn}}$, $\operatorname{diag}\left(\mathrm{R}_{11}\right)=(1, \ldots, 1), \quad \operatorname{tr}\left(\mathrm{R}_{11}\right)=1+\ldots+1=\operatorname{tr}\left(\Lambda_{11}\right)=$ $\lambda_{1}+\ldots+\lambda_{\mathrm{n}-1}=\mathrm{n}-1, \lambda_{1} \geq \ldots \geq \lambda_{\mathrm{n}-1} \geq 0$. И эту пару матриц мы должны преобразовать в другую пару матриц $\left(\mathrm{C}^{+}{ }_{11}, \Lambda^{+}{ }_{11}\right)$.

Это достигается при решении ОС3 2, изложение ее доступно в статье [14].Отличие текста описания ОС3 2 в [14] от описания ОС3 2 в настоящей статье состоит в размерности матрицы - здесь размерность матрицы $\mathrm{C}^{+}{ }_{11}$ равна $\mathrm{n}-1$, в статье [14] используется размерность $\mathrm{n}$ матрицы $\mathrm{C}^{+}{ }_{\mathrm{nn}}$.

Вне ОЗ МЛРА реализуется этап задания пары индексов $(\mathrm{k}, \mathrm{j})$ и новые значения заданных нами выделенным компонентам $\mathrm{c}_{\mathrm{kj}}=\mathrm{c}^{+}{ }_{\mathrm{kj}}, \mathrm{j}=1, \ldots, \mathrm{n}-1$, $\mathrm{k} \in\{1, \ldots, \mathrm{n}-1\}$. В постановке задачи ОС3 для ООМ МЛРА невозможно конкретизировать этот пункт.

После решения ОСЗ 2 для ООМ МЛРА в матрице $\mathrm{C}^{+} 11$ изменяются все ее элементы, кроме выше выделенных компонент со знаком плюс (+). Матрица $\mathrm{C}^{+}{ }_{11}$ содержит заданные индикаторы присутствия знаний - «весомые» значения $\left|\mathrm{c}_{\mathrm{kj}}\right|>\mathrm{c}_{\mathrm{j}}$. Как объект будущей когнитивной модели матрица $\mathrm{C}^{+}{ }_{11}$ является индикатором присутствия знаний. Она является матрицей коэффициентов комбинационных связей [12]. Обоснование большей значимости матрицы «коэффициентов комбинационных связей» [12] $\mathrm{C}^{+} 11$ при когнитивном моделировании с применением математической модели главных компонент приведено в статье[12].

Будем использовать матрицу собственных векто ров $\mathrm{C}^{+}{ }_{11}$. Для каждого ј-ого собственно вектора $\quad \mathbf{c}_{\mathbf{j}}=\left(\mathrm{c}_{1 \mathrm{j}}, \mathrm{c}_{2 \mathrm{j}} \ldots \mathrm{c}_{\mathrm{n}-1, \mathrm{j}}\right)^{\mathrm{T}}, \quad \mathrm{j}=1, \ldots, \mathrm{n}-1$. выберем пары номеров $(\mathrm{k}, \mathrm{j})$ разных компонент $\mathrm{c}_{\mathrm{kj}}$ таких, что они по абсолютной величине «весомы»: $\left|\mathrm{c}_{\mathrm{kj}}\right|>\mathrm{c}_{\mathrm{j}}$. 


\begin{tabular}{|c|c|c|c|c|c|c|}
\hline \multirow{4}{*}{ Impact Factor: } & ISRA (India) & $=3.117$ & SIS (USA) & $=0.912$ & ICV (Poland) & $=6.630$ \\
\hline & ISI (Dubai, UAI & $=0.829$ & РИНЦ (Russia & $=0.156$ & PIF (India) & $=1.940$ \\
\hline & GIF (Australia) & $=0.564$ & ESJI (KZ) & $=8.716$ & IBI (India) & $=4.260$ \\
\hline & JIF & $=1.500$ & SJIF (Morocce & $=5.667$ & OAJI (USA) & $=0.350$ \\
\hline
\end{tabular}

Пары номеров $\{(\mathrm{k}, \mathrm{j})\}$ образуют «конфигурацию», соответствующую индексам $(k, j)$ элементов $c_{k j} j$ ых собственных векторов $\mathbf{c}_{\mathrm{j}}=\left(\mathrm{c}_{1 \mathrm{j}}, \mathrm{c}_{2 \mathrm{j}} \ldots, \mathrm{c}_{\mathrm{n}-1-1, \mathrm{j}}\right)^{\mathrm{T}}$, $\mathrm{j}=1, \ldots, n-1$. Примеры выделенных по разным критериям пар номеров $(\mathrm{k}, \mathrm{j})$ разных компонент элементов $c_{\mathrm{kj}}$ приведены в статьях $[7,14,15]$. Все выделенные элементы находятся в матрице $\mathrm{C}^{+}{ }_{11}$.Полученные модельные стандартизованные значения $\mathrm{Z}_{\mathrm{n}}^{+}$изменяются относительно значений $\mathrm{z}^{+}{ }_{1}, \mathrm{z}^{+}{ }_{2}, \ldots, \mathrm{z}^{+}{ }_{\mathrm{n}-1}$ по формуле $\mathrm{z}^{+}{ }_{\mathrm{n}}=\beta_{1} \mathrm{z}^{+}{ }_{1}+\beta_{2} \mathrm{z}^{+}{ }_{2}+\ldots+\beta_{\mathrm{n}-}$ ${ }_{1} \mathrm{Z}_{\mathrm{n}-1}^{+}$, . В соответствии с разбиением $\left(\mathrm{z}_{\mathrm{i} 1}, \ldots, \mathrm{Z}_{\mathrm{i}, \mathrm{n}-1} \mid \mathrm{Z}_{\mathrm{in}}\right)$ $\mathrm{z}$-переменных $\mathrm{m}$ значений всех $\mathrm{n} \mathrm{z}$-переменных образуют 2 подматрицы $\mathrm{Z}^{+}{ }_{1}, \mathrm{Z}^{+}{ }_{2}$ матрицы для m-на$\mathrm{n}$ матрицы $\mathrm{Z}_{\mathrm{mn}}^{+}=\left[\mathrm{Z}^{+}{ }_{1} \mathrm{~J} \mathrm{Z}_{2}^{+}\right]$.

Для полной корреляционной матрицы $\mathrm{R}_{\mathrm{nn}}$, не используемой в 1МЛРА, имеются свои ортогонормированная матрица собственных векторов $\mathrm{C}_{\mathrm{nn}}$ (решение ПСЗ), согласованная со своим спектром $\Lambda_{\mathrm{nn}}=\operatorname{diag}\left(\lambda_{1}, \ldots \lambda_{\mathrm{n}}\right)$ таким образом, что выполняются равенства $\mathrm{R}_{\mathrm{nn}} \mathrm{C}_{\mathrm{nn}}=\mathrm{C}_{\mathrm{nn}} \Lambda_{\mathrm{nn}}$, $\mathrm{C}^{\mathrm{T}}{ }_{\mathrm{nn}} \mathrm{C}_{\mathrm{nn}}=\mathrm{C}_{\mathrm{nn}} \mathrm{C}^{\mathrm{T}}{ }_{\mathrm{nn}}=\mathrm{I}_{\mathrm{nn}}, \quad \operatorname{dag}\left(\mathrm{R}_{\mathrm{nn}}\right)=(1, \ldots, 1)$, $\operatorname{tr}\left(R_{n n}\right)=1+1++1=\operatorname{tr}\left(\Lambda_{n n}\right)=\lambda_{1}+\ldots+\lambda_{n}=n, \quad \lambda_{1} \geq \ldots \geq \lambda_{n} \geq 0$. Эта матрица $R_{n n}$ необходима для решения прямой задачи - ПЗ МЛРА. Решение ПЗ МЛРА даст те же матричные объекты, что и в ОЗ МЛРА.

Пусть в матрице собственных векторов $\mathrm{C}^{+} 11$ для каждого ј-ого собственно вектора $\mathbf{c}^{+}{ }_{j}=\left(\mathrm{c}^{+}{ }_{1 j}, \mathrm{c}^{+}{ }_{2 \mathrm{j}} \ldots{ }^{+} \mathrm{c}_{\mathrm{n}-1, \mathrm{j}}\right)^{\mathrm{T}}, \mathrm{j}=1, \ldots, \mathrm{n}-1$ назначены пары номеров $(\mathrm{k}, \mathrm{j})$ разных компонент $\mathrm{c}_{\mathrm{kj}}$ таких, что они по абсолютной величине «весомы»: $\left|\mathrm{c}^{+}{ }_{\mathrm{kj}}\right|>\mathrm{c}_{\mathrm{j}}$. Тогда задача ОСЗ МЛРА формулируется так.

Задача (ОСЗ МЛРА):для пары матриц $\left(\mathrm{C}_{11}, \Lambda_{11}\right)$ с назначенными значениями элементов матрицы $\mathrm{C}_{11} \mathrm{c}_{\mathrm{kj}}=\mathrm{c}^{+}{ }_{\mathrm{kj}}$ требуется найти новую пару матриц $\left(\mathrm{C}^{+}{ }_{11}, \Lambda^{+}{ }_{11}\right)$,таких что матрицы $\mathrm{C}^{+}{ }_{11}$ и $\Lambda^{+}{ }_{11}$ должны удовлетворять равенствам: $\mathrm{C}^{+\mathrm{T}}{ }_{11} \mathrm{C}^{+}{ }_{11}=\mathrm{C}^{+}{ }_{11} \mathrm{C}^{+\mathrm{T}}{ }_{11}=\mathrm{I}_{(\mathrm{n}-1)(\mathrm{n}-1),}, \quad \mathrm{C}^{+}{ }_{11} \Lambda^{+}{ }_{11} \mathrm{C}^{+\mathrm{T}}{ }_{11}=\mathrm{R}^{+}{ }_{11}$, $\lambda^{+}{ }_{1}+\ldots+\lambda^{+}{ }_{n-1}=n-1, \quad \mathbf{c}_{\mathrm{j}}{ }^{+} \Lambda^{+}{ }_{11} \mathbf{c}_{\mathrm{j}}{ }^{+\mathrm{T}}=1, \quad \mathbf{c}_{\mathrm{i}}{ }^{+} \Lambda^{+}{ }_{11} \mathbf{c}_{\mathrm{j}}{ }^{+\mathrm{T}}=\mathrm{r}^{+}{ }_{\mathrm{ij}}$, $\mathrm{r}^{+}{ }_{\mathrm{ji}}=\mathrm{r}^{+}{ }_{\mathrm{ij}}, \mathrm{j}=1, \ldots, \mathrm{n}-1 . \quad$ где вновь определяемая корреляционная матрица $\mathrm{R}^{+}{ }_{11}=\mathrm{C}^{+}{ }_{11} \Lambda^{+}{ }_{11} \mathrm{C}^{+\mathrm{T}}{ }_{11}$, имеет новые матрицы собственных векторов $\mathrm{C}^{+}{ }_{11}$ и собственных чисел $\Lambda^{+}{ }_{11}=\operatorname{diag}\left(\lambda^{+}{ }_{1}, \ldots, \lambda^{+} \mathrm{n}-1\right)$, $\lambda^{+}{ }_{1}+\ldots+\lambda^{+}{ }_{\mathrm{n}-1}=\mathrm{n}-1, \lambda^{+}{ }_{1} \geq \ldots \geq \lambda^{+}{ }_{\mathrm{n}-1}$.

При этом матрица $\mathrm{C}^{+}{ }_{11}$ имеет те же заданные значения $\mathrm{c}^{+}{ }_{\mathrm{kj}}$ компонентов $\mathrm{c}^{+}{ }_{\mathrm{kj}}, \mathrm{j}=1, \ldots, \mathrm{n}-1$, $\mathrm{k} \in\{1, \ldots, \mathrm{n}-1\}$ у $\mathrm{n}-1$ собственных векторов $\mathbf{c}^{+}=\left(\mathrm{c}^{+}{ }_{1 \mathrm{j}}, \mathrm{c}^{+}{ }_{2 \mathrm{j}} \ldots \mathrm{c}^{+}{ }_{\mathrm{n}-1, \mathrm{j}}\right)^{\mathrm{T}}$, расположенных по столбцам матрицы $\mathrm{C}^{+}{ }_{11}=\left[\mathbf{c}_{1}^{+}\left|\mathbf{c}^{+}\right| \ldots \mid \mathbf{c}^{+}{ }_{\mathrm{n}-1}\right]$.

В результате решения ОСЗ для ООМ МЛРА вычисляются 2 матрицы: ортогональная матрица $\mathrm{C}^{+}{ }_{11}$ собственных векторов $\mathbf{c}^{+}{ }_{\mathbf{j}}=\left(\mathrm{c}^{+}{ }_{1 \mathrm{j}}, \mathrm{c}^{+}{ }_{2 \mathrm{j}} \ldots \mathrm{c}^{+}{ }_{\mathrm{n}-1, \mathrm{j}}\right)^{\mathrm{T}}$, расположенных по её столбцам: $\mathrm{C}^{+}{ }_{11}=\left[\mathbf{c}^{+}\left|\mathbf{c}^{+}{ }_{2}\right| \ldots \mid \mathbf{c}^{+}{ }_{n-1}\right]$ и согласованная со спектром $\Lambda_{(\mathrm{n}-1)(\mathrm{n}-1)}=\operatorname{diag}\left(\lambda^{+}{ }_{1}, \ldots \lambda^{+}{ }_{\mathrm{n}-1}\right)$ таким образом, что выполняются соотношения $\mathrm{R}^{+}{ }_{11} \mathrm{C}^{+}{ }_{11}=\mathrm{C}^{+}{ }_{11} \Lambda^{+}{ }_{11}$, $\mathrm{C}^{+\mathrm{T}}{ }_{11} \mathrm{C}^{+}{ }_{11}=\mathrm{C}_{11} \mathrm{C}^{\mathrm{T}}{ }_{11}=\mathrm{I}_{\mathrm{nn}}, \quad \operatorname{diag}\left(\mathrm{R}^{+}{ }_{11}\right)=(1, \ldots, 1)$,
$\operatorname{tr}\left(\mathrm{R}^{+}{ }_{11}\right)=1+\ldots+1=\operatorname{tr}\left(\Lambda_{11}\right)=\lambda_{1}+\ldots+\lambda_{\mathrm{n}-1}=\mathrm{n}-1, \quad \lambda_{1} \geq \ldots \geq$ $\lambda_{\mathrm{n}-1} \geq 0$.

Если будем решать ПСЗ при когнитивном извлечении заложенных нами знаний, то решениями ПСЗ в нашем примере являются 2 матрицы $\mathrm{C}_{66}^{+}, \quad \Lambda_{66}^{+}, \quad \mathrm{a}$ ортогональное преобразование - матрица $\mathrm{C}^{+} 66$, примененное к матрице $\mathrm{z}$-переменных $\mathrm{Z}_{1}$.

В модель ОСЗ для ООМ МЛРА заложено выполнение условия равенства произведения матриц $\mathrm{C}^{+}{ }_{11} \Lambda^{+}{ }_{11} \mathrm{C}^{+\mathrm{T}}{ }_{11}$ матрице $\mathrm{R}^{+}{ }_{11}$ коэффициентов корреляции: $\mathrm{C}^{+}{ }_{11} \Lambda^{+}{ }_{11} \mathrm{C}^{+\mathrm{T}}{ }_{11}=\mathrm{R}^{+}{ }_{11}$. Это условие присутствует в программе-таблице модели ОС3 для ООМ МЛРА (в процедуре Solver) в виде функций ограничений для целевой функции вида $\lambda^{+}{ }_{1}+\ldots+\lambda^{+}{ }_{6}=6$.

Функции ограничений для целевой функции имеют вид $\mathbf{c}_{\mathrm{j}}^{+} \Lambda^{+}{ }_{66} \mathbf{c}_{\mathrm{j}}{ }^{+\mathrm{T}}=1, \mathbf{c}_{\mathrm{i}}^{+} \Lambda^{+}{ }_{66} \mathbf{c}_{\mathrm{j}}{ }^{+\mathrm{T}}=\mathrm{r}_{\mathrm{ij}}^{+}, \mathrm{r}_{\mathrm{ji}}{ }^{+}=\mathrm{r}_{\mathrm{ij}}^{+}$, $\mathrm{j}=1, \ldots, 5$. Неизвестными переменными являются элементы матрицы $\mathrm{C}^{+} 11$ и наличие для нее начальных элементов в модели ОС3 2для ООМ МЛРА обязательно.

Поэтому модель ОСЗ для ООМ МЛРА использует полную матрицу $\mathrm{C}^{+(\ell)}$. При этом эти матрицы собственных векторов не должны иметь одну и ту же матрицу собственных чисел. Решение оптимизационной задачи (смотрите ниже) обеспечивает моделирование матрицы $\Lambda^{+} 66$ как матрицы собственных чисел, а матрицу $\mathrm{C}_{66}^{+}-$ как матрицы собственных векторов для не нужной нам корреляционной матрицы $\mathrm{R}^{+}{ }_{66}$. А не любой симметрической матрицы. Условие, налагаемое на тип матрицы $\mathrm{R}^{+}{ }_{11}$ требует ввода в соотношения модели ОСЗ для ООМ МЛРА дополнительных ограничений на ее элементы. Эти ограничения существенны в решаемой оптимизационной задаче.

Мы рассматриваем случай $\mathrm{n}=6$ из-за использования нами разных «конфигураций», образуемых индексами $(\mathrm{k}, \mathrm{j})$ элементов $\mathrm{c}_{\mathrm{kj}} \mathrm{j}$-ых собственных векторов $\mathbf{c}_{\mathbf{j}}=\left(\mathrm{c}_{1 \mathrm{j}}, \mathrm{c}_{2 \mathrm{j}} \ldots \mathrm{c}_{\mathrm{n}-1, \mathrm{j}}\right)^{\mathrm{T}}, \mathrm{j}=1, \ldots, 6$. $\mathrm{B}$ разных матрицах $\mathrm{C}_{66}$ мы выделяли по разным критериям пары номеров $(\mathrm{k}, \mathrm{j})$ разных компонент элементов $\mathrm{c}_{\mathrm{kj}}$. Bce элементы находятся в подматрице $\mathrm{C}^{+}$.

Решения $\mathrm{C}^{+}{ }_{11}$ ОС 3 для ООМ МЛРА существенно облегчают задачи моделирования пар матриц $\left(\mathrm{C}^{+}{ }_{11}, \Lambda^{+}{ }_{11}\right)$, удовлетворяющих тому или иному критерию $[1,5-7]$ и соотношениям $\mathrm{C}^{+\mathrm{T}}{ }_{11} \mathrm{C}^{+}{ }_{11}=\quad \mathrm{C}^{+}{ }_{11} \mathrm{C}^{+\mathrm{T}}{ }_{11}=\mathrm{I}(\mathrm{n}-1)(\mathrm{n}-1), \quad \mathrm{R}^{+}{ }_{11} \mathrm{C}^{+}{ }_{11}=\mathrm{C}^{+}{ }_{11} \Lambda^{+}{ }_{11}$, $\mathrm{R}^{+\mathrm{T}}{ }_{11}=\mathrm{R}^{+}{ }_{11}$.

Матрица $\mathrm{C}_{11}$ имеет весомые значения, являющиеся выделенными компонентами собственных векторов $\left[\mathbf{c}_{1}|\ldots| \mathbf{c}_{\mathbf{n}-1}\right]$. Выделенные компоненты присутствовали в ПСЗ (в матрице $\mathrm{C}_{66}$ ) и активно участвовали при извлечении 


\begin{tabular}{|c|c|c|c|c|c|c|}
\hline \multirow{4}{*}{ Impact Factor: } & ISRA (India) & $=3.117$ & SIS (USA) & $=0.912$ & ICV (Poland) & $=6.630$ \\
\hline & ISI (Dubai, UAI & $=0.829$ & РИНЦ (Russia & $=0.156$ & PIF (India) & $=1.940$ \\
\hline & GIF (Australia) & $=0.564$ & ESJI (KZ) & $=8.716$ & IBI (India) & $=4.260$ \\
\hline & JIF & $=1.500$ & SJIF (Morocce & $=5.667$ & OAJI (USA) & $=0.350$ \\
\hline
\end{tabular}

цифровых знаний [1,5-7], где подматрица $\mathrm{C}_{11}$ была единственно доступной.

Для каждой матрицы $\mathrm{C}^{(\ell)}{ }_{11}$-решения ОС3 1 вида $\Lambda_{11}=>\left(\mathrm{C}^{(\ell)}{ }_{11} \mathrm{R}^{(\ell)}{ }_{11}\right), \ell=1, \ldots, \mathrm{k}_{\ell}=500$ решение матрица $\mathrm{C}^{+} 11$ нашей ОСЗ для ООМ МЛРА, моделирует множество других матриц $\mathrm{C}^{+(\ell)}{ }_{11}$, каждая из них является источником косвенных сведений и «выдает» те же цифровые знания [1,57] что были «заложены» в матрицу $\mathrm{C}_{11}$ или $\mathrm{C}^{+}{ }_{11}$. Одни и те же знания соответствуют нашему множеству цифровых данных, адекватных реальным данным (таблицам «объект-свойства»). В данной статье ОСЗ для ООМ МЛРА решается после решения ПСЗ, так как в работах [1,5-7] при извлечении цифровых знаний применялась ПМ ГК. Но можно использовать и решение ОСЗ 1. Случаев необходимости использования решений ОС3 1 существует много. Здесь их не рассматриваем.

Программа -таблица моделирования матрицы $\mathrm{C}^{(+)} 11$ и матрицы $\Lambda^{+}{ }_{11}$

Описание нашей программы повторяет описание программы -таблицы из статьи [1]. Только размерность у нас $\mathrm{n}-1=5$ а не $\mathrm{n}=6$. В [1] моделируется матрица $\mathrm{R}^{(+)}{ }_{n n}$, здесь же мы излагаем программу моделирования матрицы $\mathrm{C}^{(+)}{ }_{11}$ и матрицы $\Lambda^{+}{ }_{11}$. Их размерности равны $(\mathrm{n}-1)$ -на-(n-1).

Введем в ячейки ЭТ Excel известные значения элементов матриц $\mathrm{C}_{11}$ и $\Lambda_{11}$ [1]. Проверяем в программе-таблице (Таблица 2) выполнение и точность равенств $\mathrm{C}^{\mathrm{T}}{ }_{11} \mathrm{C}_{11}=\mathrm{C}_{11} \mathrm{C}^{\mathrm{T}}{ }_{11}=\mathrm{I}_{(\mathrm{n}-1)(\mathrm{n}-1),}, \quad \mathrm{R}_{11} \mathrm{C}_{11}=\mathrm{C}_{11} \Lambda_{11} . \quad$ Эти значения являются начальными для реализации метода GRD2 в процедуре Solver. Далее реализуем ниже приведенные Шаги 1-7.

Шаг 1. Выделяем индексы $(\mathrm{k}, \mathrm{j})$ и значения элементов $\mathrm{c}_{\mathrm{kj}}$ из подматрицы $\mathrm{C}_{1}$ с выделенными индексами Так как $\ell=5$, о введем пары ячеек ЭТ Excel (имени и значения элементов) из каждого столбца матрицы $\mathrm{C}_{66}^{+}$. Эти элементы не изменяют своих значений.

Шаг 2. Ввод в ячейки ЭТ Excel скалярных равенств, взятых из матричных равенств $\mathrm{C}^{+\mathrm{T}}{ }_{55} \mathrm{C}^{+}{ }_{55}=\mathrm{C}^{+}{ }_{55} \mathrm{C}^{+\mathrm{T}}{ }_{55}=\mathrm{I}_{55}, \quad \mathrm{R}^{+}{ }_{55}=\mathrm{C}^{+}{ }_{55} \Lambda^{+}{ }_{55} \mathrm{C}^{+\mathrm{T}}{ }_{55} . \quad$ Для элементов моделируемого нового спектра ${ }_{66} \Lambda^{+}{ }_{66}=\operatorname{diag}\left(\lambda^{+}{ }_{1}, \ldots, \lambda^{+}{ }_{6}\right) \quad$ введем $\lambda^{+}{ }_{1}+\ldots+\lambda^{+}{ }_{5}=5, \quad \lambda^{+}{ }_{1}>\ldots>\lambda^{+}{ }_{5}>0, \quad \lambda^{+}{ }_{1}=\lambda_{1}, \quad$ где $\lambda_{1-}$ известный всегда наибольший элемент известного спектра $\Lambda_{55}=\operatorname{diag}\left(\lambda_{1}, \ldots, \lambda_{5}\right)$.

Шаг 3. Назначить ячейку с формулой $\lambda^{+}{ }_{1}+\ldots+\lambda^{+}{ }_{5}$ целевой функцией процедуры Solver.

Шаг 4. Назначить в качестве изменяемых ячеек (неизвестных переменных задачи) ячейки матрицы $\mathrm{C}_{55}$ и ячейки элементов столбцов №1,...№5 матрицы $\mathrm{C}^{+}{ }_{55}$.

Этим действием достигается неизменяемость компонентов собственных векторов №1, .., №5.

Шаг 5. Назначить в качестве изменяемых еще 5 ячеек с значениями $\lambda^{+}{ }_{1} \ldots, \lambda^{+}{ }_{5}$.

Шаг 6. Ввод в строке окна Solver ограничений для ячеек с их значениями вида $\lambda^{+}{ }_{1} \ldots>0, \ldots, \lambda^{+}{ }_{6}>0, \lambda^{+}{ }_{1}=\lambda_{1}$.

Шаг 7. Нажать кнопку «Выполнить».

Моделируем нужную нам пару матриц $\left(\mathrm{C}^{+}{ }_{11}, \Lambda^{+}{ }_{11}\right)$. Они определяют матрицу $\mathrm{R}^{+}{ }_{11}=\mathrm{C}^{+}{ }_{11} \Lambda^{+}{ }_{11} \mathrm{C}^{+\mathrm{T}}{ }_{11}$. Следовательно, вместо схемы $\left(\mathrm{n}, \varphi_{11}\right)=>\left(\mathrm{R}_{11}\right)$ мы реализовали схему $\left(\mathrm{n}, \varphi_{11}\right)=>\left(\mathrm{R}^{+}{ }_{11}\right)$. Чтобы вычислить требуемую матрицу $\mathrm{R}^{(+)}{ }_{12}$ нужно умножить известный вектор коэффициентов регрессии $\beta$ на модельную матрицу $\mathrm{R}^{(+)}{ }_{11}: \mathrm{R}^{(+)}{ }_{12}=\mathrm{R}^{(+)}{ }_{11} \beta$.

Этот новый вектор $\beta^{(+)}=\mathrm{R}^{+-1}{ }_{11} \mathrm{R}^{+}{ }_{12}$ отличается от старого вектора $\beta=\mathrm{R}^{-1}{ }_{11} \mathrm{R}_{12}$, тем, что его компоненты зависят от матрицы $\mathrm{C}^{+}{ }_{11}$, содержащей нами введенные знания. Индикаторы присутствия знаний были заложены в нее. Зависящая от матрицы $\mathrm{C}^{+} 11$ корреляционная матрица $\mathrm{R}^{+}{ }_{11}=\mathrm{C}^{+}{ }_{11} \Lambda^{+}{ }_{11} \mathrm{C}^{+\mathrm{T}}{ }_{11} \quad$ является $\mathrm{C}^{+}{ }_{11}$-источником знаний. Формула вектора для вектора $\boldsymbol{\beta}$ в ООМ МЛРА принимает вид $\beta=\left(\mathrm{R}^{+}{ }_{11}, \mathrm{R}^{+}{ }_{12}=\right.$ $\mathrm{C}^{+}{ }_{11} \Lambda^{+}{ }_{11} \mathrm{C}^{+\mathrm{T}}{ }_{11} \mathrm{R}^{+}{ }_{12}$ изобразим так: $\left(\mathrm{R}^{+}{ }_{11}, \mathrm{C}^{+}{ }_{11}, \Lambda^{+}{ }_{11}\right)=>$ $\left(\mathrm{Y}^{(\mathrm{t})}{ }_{\mathrm{m}(\mathrm{n}-1)}, \mathrm{Z}^{(\mathrm{t})} \mathrm{m}(\mathrm{n}-1)\right), \mathrm{t}=1, \ldots, \mathrm{K}_{\mathrm{t}}<\infty$, а подзадачу 3 $\left(\mathrm{R}^{+}{ }_{11}, \beta\right)=>\mathrm{R}^{+}{ }_{12}$. Результат решений трех подзадач обозначается в виде: $\left(\mathrm{m}, \mathrm{n}, \varphi_{11}, \boldsymbol{\beta}, \mathrm{C}^{+}{ }_{11}\right)=>$ $\left(\mathrm{R}^{+}{ }_{11}, \mathrm{R}^{+}{ }_{12}, \mathrm{Z}^{+}{ }_{1}, \mathrm{Z}^{+}{ }_{2}\right)$.

Практические рекомендации по эксплуатации программы-таблицы для решения подзадачи 1.

Шаг 1. При фиксированном значении параметра $\varphi_{11}$ моделируем спектр $\Lambda_{\mathrm{n}-1)(\mathrm{n}-1)}=$ $\operatorname{diag}\left(\lambda_{1}, \ldots, \lambda_{\mathrm{n}-1}\right)$, с значением $\varphi$-параметра спектра $\Lambda_{(\mathrm{n}-1)(\mathrm{n}-1)} \varphi=\left[\left(\mathrm{f}_{2}\left(\Lambda_{(\mathrm{n}-1)(\mathrm{n}-1)}\right)-\mathrm{n}\right) /(\mathrm{n}(\mathrm{n}-1)]^{1 / 2}, \quad\right.$ равного заданному значению $\varphi_{11}$. Значение $\varphi_{11}$ должно принадлежать одному из 5 интервалов изменения коэффициента корреляции по шкале Чеддока (Chaddock scale). По этой шкале количественная мера тесноты связи: абсолютное значение коэффициента корреляции, принадлежащее интервалу от 0 до 0.3 - качественно интерпретируется как «очень слабая», интервалу от 0.3 до 0.5-«слабая, умеренная», интервалу от 0.5 до 0.7-«заметная» (moderate positive), интервалу от 0.7 до 0.9-«высокая», интервалу от 0.9 до 1-«очень высокая». Схему этого Шага 1 обозначим так: $\left(\mathrm{n}-1, \varphi_{11}\right)=>\left\{\Lambda_{\mathrm{n}-1)(\mathrm{n}-1)}=\operatorname{diag}\left(\lambda_{1}, \ldots, \lambda_{\mathrm{n}-1}\right)\right\} . \quad$ Фигурные скобки \{\} обозначают бесконечность множества таких спектров. При этом элементы $\lambda_{1}, \ldots, \lambda_{\mathrm{n}-1}$ спектра $\Lambda_{\mathrm{n}-1)(\mathrm{n}-1)}$ неизвестной корреляционной матрицы $\mathrm{R}_{\mathrm{n}-1)(\mathrm{n}-1)}$ моделируем с применением программы SPEKTR из ППП «Спектр» [10]. 


\begin{tabular}{llllll} 
& ISRA (India) $=\mathbf{3 . 1 1 7}$ & SIS (USA) $=\mathbf{0 . 9 1 2}$ & ICV (Poland) & $\mathbf{= 6 . 6 3 0}$ \\
Impact Factor: & ISI (Dubai, UAE) $=\mathbf{0 . 8 2 9}$ & PUHЦ (Russia) $=\mathbf{0 . 1 5 6}$ & PIF (India) & $=\mathbf{1 . 9 4 0}$ \\
& GIF (Australia) $=\mathbf{0 . 5 6 4}$ & ESJI (KZ) & $\mathbf{8 . 7 1 6}$ & IBI (India) & $\mathbf{4 . 2 6 0}$ \\
& JIF & $\mathbf{1 . 5 0 0}$ & SJIF (Morocco) $=\mathbf{5 . 6 6 7}$ & OAJI (USA) & $\mathbf{= 0 . 3 5 0}$ \\
\hline
\end{tabular}

Заметим, что программа SPEKTR не моделирует матрицу $\mathrm{R}_{\mathrm{n}-1)(\mathrm{n}-1)}$ (в виде блока $\mathrm{R}_{11}$ ) а моделирует только спектр $\Lambda_{\mathrm{n}-1)(\mathrm{n}-1)}=\operatorname{diag}\left(\lambda_{1}, \ldots, \lambda_{\mathrm{n}-1}\right)$ с заданным значением f-параметра $\mathrm{f}_{2}\left(\Lambda_{\mathrm{n}-1)(\mathrm{n}-1)}\right)=\lambda^{2}{ }_{1}+\ldots+\lambda^{2}$ ${ }_{1}=\mathrm{f}_{2}\left(\mathrm{R}_{\mathrm{n}-1)(\mathrm{n}-1)}\right)$. Элементы спектра упорядочены в порядке убывания: $\lambda_{1}>\ldots>\lambda_{\mathrm{n}-1}>0, \lambda_{1}+\ldots+\lambda_{\mathrm{n}-1}=\mathrm{n}-1$, при чем матрица $\Lambda_{\mathrm{n}-1)(\mathrm{n}-1)}$ может быть как полноранговой, так и, при необходимости, может быть смоделирован и с неполным рангом. Тогда число ее положительных элементов равно $\mathrm{k}<\mathrm{n}-1$. Одна из таблиц значений $\varphi_{11}$, принадлежащих 5 интервалам изменения параметра $\varphi$, приведена в статье [9, стр.187, Таблица 1].

Приведенные «Модельные значения элементов 20 спектров 20 неизвестных корреляционных матриц, имеющих заданные значения f-параметров спектра» получены при значениях

$\varphi_{11}=0.20,0.35,0.40,0.45,0.50,0.55,0.60,0.65,-0.70$, $0.70,0.75,0.80,0.80,0.85,0.85,0.90,0.90,0.95,0.98$.

Значение $\varphi_{11}\left(0<\varphi_{11}<1\right)$ не зависит от значения $\mathrm{n}$. Оно позволяет моделировать n-1 положительных элементов $\lambda_{1}, \ldots, \lambda_{\mathrm{n}-1}$, в сумме равных n- 1 . Значение $\varphi_{11}$ регулирует интервал значений, в пределах которого изменяются значения генерируемых коэффициентов из матрицы $\mathrm{C}^{+}{ }_{11}$. Примеры фиксации интервалов для значений $\varphi_{11}$ приведены, например, в [9,стр.187].

Спектр $\Lambda_{\mathrm{n}-1)(\mathrm{n}-1)}$ может быть получен и подругому. Если требуется моделировать спектр с заданными значениями его $f$-параметров $\mathrm{f}_{1}, \mathrm{f}_{2}, \mathrm{f}_{4}$, то задача моделирования $\left(\mathrm{f}_{1}, \mathrm{f}_{2}, \mathrm{f}_{4}\right)$-спектров $\Lambda_{\mathrm{n}-1)(\mathrm{n}-1)}$ $\left(f_{1}=n-1\right)$ по-разному решены в [4-11]. Bce модельные $\left(\mathrm{f}_{1}, \mathrm{f}_{2}, \mathrm{f}_{4}\right)$-спектры из их бесконечного множества имеют заданные значения $\mathrm{f}$ параметров $\mathrm{f}_{2}, \mathrm{f}_{4}, \mathrm{c}$ заданной погрешностью $[1,2]$ $\left\|\mathrm{f}_{2}-\mathrm{f}_{2}\left(\Lambda_{\mathrm{nn}}\right)\right\| \leq \mathrm{e}_{0},\left\|\mathrm{f}_{4}-\mathrm{f}_{4}\left(\Lambda_{\mathrm{nn}}\right)\right\|=0$.

Шаг 2. Моделирование блока $\mathrm{R}_{11}$ корреляционной матрицы $\mathrm{R}_{\mathrm{nn}}$. Здесь нам предстоит воспользоваться одним из решений из бесконечного множества решений Обратной спектральной задачи (ОС3): $\Lambda_{\mathrm{n}-1)(\mathrm{n}-1)}=>\left(\mathrm{C}^{(\ell)} \mathrm{n}_{-1)(\mathrm{n}-1)}\right.$, $\left.\mathrm{R}^{(\ell)}{ }_{\mathrm{n}-1)(\mathrm{n}-1)}\right), \quad \ell=1, \ldots, \mathrm{k}_{\ell}<\infty . \quad$ Здесь мы будем использовать одну из корреляционных матриц $\mathrm{R}^{(\ell)}{ }_{\mathrm{n}-1)(\mathrm{n}-1)}$, элементы которой по абсолютным величинам должны удовлетворять нас и соответствовать выбранному значению $\varphi_{11}$ в предыдущем Шаге 1.

Для моделирования бесконечного множества корреляционных матриц $\mathrm{R}_{\mathrm{n}-1)(\mathrm{n}-1)}$ с одним и тем же спектром $\quad \Lambda_{\mathrm{n}-1)(\mathrm{n}-1)}=\operatorname{diag}\left(\lambda \quad 1, \ldots, \lambda_{\mathrm{n}-1}\right) \quad$ используем программу CORMAP (CORMAT, COMA31) из ППП «Спектр» [10]. В нашей ОЗ МЛРА и в других задачах анализа данных, где применяется ОМ ГК [2-5], для модельного спектра $\Lambda_{(\mathrm{n}-1)(\mathrm{n}-1)}$ существует бесконечное множество корреляционных матриц $\mathrm{R}_{(\mathrm{\ell})(\mathrm{n})(\mathrm{n}-1), \ell=1, \ldots, \mathrm{K}_{\ell}<\infty}$ (лемма C.P.Chalmers [12]).
Зафиксируем номер $\ell$ решения обратной спектральной задачи (ОС3) [5], а полученную корреляционную матрицу $\mathbf{R}^{(\ell)}{ }_{n-1)(n-1)}$ обозначим как искомый блок $\mathrm{R}_{11}$ матрицы $\mathrm{R}_{\mathrm{nn}}$. Подзадача 1 : $\left(\mathrm{n}, \varphi_{11}\right)=>\left(\mathrm{R}^{(\ell)}{ }_{11}\right), \ell=1, \ldots, \mathrm{K} \ell<\infty$ решена.

Необходимо проверять точность равенства левой части формулы ее правой части во всех матричных равенствах нашей модели. Например, по условию ООЗ МЛРА элементы из подматрицы $\mathrm{R}^{(\ell)}{ }_{12}=\mathrm{R}^{(\ell)}{ }_{11} \beta$ должны удовлетворять равенству $\mathrm{R}^{(\ell)}{ }_{12}=(1 / \mathrm{m}) \mathrm{Z}^{(\ell) \mathrm{T}}{ }_{1} \mathrm{Z}_{2}$. Это матричное уравнение должно точно выполняться численно.

Таблицы числовых значений всех матриц демонстрируют все матричные равенства. Эти же таблицы участвуют при обратном процессе извлечения введенных знаний с применением когнитивного моделирования. Совпадение элементов здесь смоделированных с элементами таблиц, полученных при когнитивном моделировании извлечения введенных знаний будет показано в другой статье.

\section{Заключение}

Разработанная модель ОСЗ для ООМ МЛРА применялась при моделировании матрицы собственных векторов и матрицы собственных чисел. Но с заданными значениями их выделенных компонент из матрицы собственных векторов. Наша модель ООМ МЛРА является вкладом в теорию обратных спектральных задач [14]. Для 5 объектов ОМ ГК в качестве входных объектов отобраны следующие полезные для пользователя подмодели [14]: $(\mathrm{R})=>,(\mathrm{Y})=>,(\mathrm{Z})=>$, $(\Lambda, C)=>,(\Lambda, R)=>,(\Lambda, Y)=>,(\Lambda, Z)=>,(\Lambda, C, R)=>$, $(\Lambda, R, Y)=>,(\Lambda, Y, Z)=>,(\Lambda, C, R, Y)=>,(\Lambda, R, Y, Z)=>$, $\mathrm{C}_{66}=\left[\mathrm{C}^{+}{ }_{1} \mid \mathrm{C}_{2}\right]=>\left(\mathrm{C}^{+}{ }_{\mathrm{nn}}, \Lambda^{+}{ }_{\mathrm{nn}}\right)=>\left(\mathrm{R}^{+}{ }_{\mathrm{nn}}, \mathrm{Z}^{(\mathrm{t})}{ }_{\mathrm{mn}}, \mathrm{Y}^{(\mathrm{t})}{ }_{\mathrm{mn}}\right)$, $\mathrm{t}=1, \ldots, \mathrm{k}_{\mathrm{t}}<\propto$, где слева от стрелки $=>$ обозначены входные объекты из ОМ ГК, а справа от стрелки $=>$ подразумевается присутствие обозначений выходных объектов из ОМ ГК. Все схемы обратных подмоделей реализованы программно.

Задачи решены с применением ООЗ АГК: $\Lambda_{11}=>\left(\mathrm{R}^{(\ell)}{ }_{11}, \mathrm{C}^{(\ell)}{ }_{11} \mathrm{Y}^{(\mathrm{t})}{ }_{\mathrm{m}(\mathrm{n}-1)}, \mathrm{Z}^{(\mathrm{t}, \ell)}{ }_{\mathrm{m}(\mathrm{n}-1)}\right), \quad \ell=1, \ldots, \mathrm{k}_{\ell}<\infty$, $\mathrm{t}=1, \ldots, \mathrm{k}_{\mathrm{t}}<\propto$. ОСЗ для ООМ МЛРА использует косвенные сведения, содержащиеся в матрице собственных векторов $\mathrm{C}^{+}{ }_{11}$, обнаруживаемые, например, при когнитивном анализе реальных данных. Применение модели ОСЗ для ООМ МЛРА в задачах моделирования цифровизации валидных и измеряемых показателей предприятия [15] актуально из-за периодического появления дополнительных сведений, отличающихся от тех, что были учтены в момент времени извлечения цифровых знаний.

Эти исследования повышают эффективность применения наших моделей и позволяя нам 


\begin{tabular}{|c|c|c|c|c|c|c|}
\hline \multirow{4}{*}{ Impact Factor: } & ISRA (India) & $=3.117$ & SIS (USA) & $=0.912$ & ICV (Poland) & $=6.630$ \\
\hline & ISI (Dubai, UAI & $=0.829$ & РИНЦ (Russia & $=0.156$ & PIF (India) & $=1.940$ \\
\hline & GIF (Australia) & $=0.564$ & ESJI (KZ) & $=8.716$ & IBI (India) & $=4.260$ \\
\hline & JIF & $=1.500$ & SJIF (Morocce & $=5.667$ & OAJI (USA) & $=0.350$ \\
\hline
\end{tabular}

перейти к решению других, более интересных задач.

\section{References:}

1. Zhanatauov, S. U. (2018). Inverse model of multiple linear regression analysis. ISJ Theoretical \& Applied Science, №4(60), pp.201212. www.t-science.org

2. Zhanatauov, S. U., \& Akhmetov, K. A. (2018). Simulation of multidimensional sample with the assigned values of the coefficients of linear regression. ISJ "Theoretical \&Applied Science". 2018, № 9(65), pp.301-314. www.t-science.org

3. Zhanatauov, S. U. (2012). Primenenie metoda lineynoy regressii dlya samootsenki riska bankrotstva banka. Finansovaya analitika: problemy i resheniya, 15(105), aprel', pp.40-43.

4. Zhanatauov, S. U. (2013). Kognitivnaya karta i model' sotsial'no-ekonomicheskikh faktorov kar'ernoy uspeshnosti shkol'nikov munitsipal'nykh shkol SShA. Sibirskiy pedagogicheskiy zhurnal, №6, pp.28-33.

5. Zhanatauov, S. U. (2014). Analiz budushchikh debitorskoy i kreditorskoy zadolzhen nostey munitsipalitetov gorodov. Ekonomicheskiy analiz:teoriya i praktika. Moscow, №2(353), pp.54-62.

6. Zhanatauov, S. U. (2015, May 16-17). Kognitivnaya karta i kognitivnaya model' analiza glavnykh komponent (telekommunikatsionnaya otrasl'). Natsional'naya assotsiatsiya uchenykh (NAU).IX Mezhd. nauch.-prakt. konf: «Otechestvennaya nauka $\mathrm{v}$ epokhu izmeneniy: postulaty proshlogo i teorii novogo vremeni». (pp.55-58). Rossiya, Ekaterinburg.

7. Zhanatauov, S. U. (2018). Modeling eigenvectors with given the values of their indicated components. Int. Scientific Journal Theoretical \&Applied Science, №11(67), pp.107-119. www.t-science.org

8. Zhanatauov, S. U. (2016). Model and histogram to adequacy of variables $(\mathrm{C}, \Lambda)$-samples and real multidimensional sample. International Scientific Journal Theoretical \&Applied Science, № 11, vol.43, pp.53-61. www.TScience.org
9. Zhanatauov, S. U. (2014). The (C,1,Y)-sample is adequate to real multidimensional sample. Proced. Int. conf.. "Leadership in Education, Business and Culture". 25 apriel 2014,AlmatySeatle,ICET USA.Leadership Iternational Conference "Leadership on Education, Business and Culture». pp.151-155.

10. Zhanatauov, S. U. (2017). Modelirovanie mnogomernykh vyborok znacheniy priznakov zernovoy kul'tury. "II mezhdun. nauchnoprakt.konf. «Evropa i tyurkskiy mir: nauka, tekhnika i tekhnologii".Izmir (Turtsiya), 29-31 maya 2017. www.regionacadem.org

11. Zhanatauov, S. U., \& Agaev, A. (2019). A giving realism to the property values of the cereal crops in the model $\Lambda$-sample stat'ya Int. Scientific Journal Theoretical \&Applied Science, №5(73), pp.101-112. www.t-science.org

12. Zhanatauov, S. U. (2019). A matrix of values the coefficients of combinational proportionality. Int. Scientific Journal Theoretical \&Applied Science, 71№3 (68), pp.401-419. www.tscience.org

13. Chalmers, C. P. (1975). Generation of correlation matrices with a given eigen structure. J. Stat. Comp. Simul., vol.4, pp.133139.

14. Zhanatauov, S. U. (2018). Inverse spectral problem with indicated values of components of the eigenvectors. ISJ Theoretical \&Applied Science, №11(67), pp.359-370. www.tscience.org

15. Zhanatauov, S. U. (2018). Inverse spectral problem. ISJ Theoretical \&Applied Science, №12(68).

16. Zhanatauov, S. U. (2017). The optimization problem with linearized equations f-parameters (f1, $\mathrm{f2}, \mathrm{f3}, \mathrm{f} 4, \mathrm{f5}, \mathrm{f} 6)$-spectrum. International scientific journal Theoretical \&Applied Science, №11, vol.55, pp.251-267. www.t-science.org 